\title{
A Novel Construction of Substitution Box Involving Coset Diagram and a Bijective Map
}

\author{
Abdul Razaq, ${ }^{1}$ Awais Yousaf, ${ }^{2}$ Umer Shuaib, ${ }^{3}$ Nasir Siddiqui, ${ }^{4}$ \\ Atta Ullah, ${ }^{5}$ and Adil Waheed ${ }^{6}$ \\ ${ }^{1}$ Department of Mathematics, University of Education Lahore, Jauharabad Campus, Jauharabad, Pakistan \\ ${ }^{2}$ Department of Mathematics, The Islamia University of Bahawalpur, Bahawalpur, Pakistan \\ ${ }^{3}$ Department of Mathematics, Government College University Faisalabad, Faisalabad, Pakistan \\ ${ }^{4}$ Department of Basic Sciences, University of Engineering and Technology, Taxila, Punjab, Pakistan \\ ${ }^{5}$ Department of Mathematics, Quaid-i-Azam University, Islamabad, Pakistan \\ ${ }^{6}$ Department of Information Technology, University of Education Lahore, Jauharabad Campus, Jauharabad, Pakistan
}

Correspondence should be addressed to Abdul Razaq; makenqau@gmail.com

Received 15 August 2017; Accepted 10 October 2017; Published 20 November 2017

Academic Editor: Zheng Yan

Copyright (C) 2017 Abdul Razaq et al. This is an open access article distributed under the Creative Commons Attribution License, which permits unrestricted use, distribution, and reproduction in any medium, provided the original work is properly cited.

The substitution box is a basic tool to convert the plaintext into an enciphered format. In this paper, we use coset diagram for the action of $\operatorname{PSL}(2, \mathbb{Z})$ on projective line over the finite field $G F\left(2^{9}\right)$ to construct proposed S-box. The vertices of the cost diagram are elements of $G F\left(2^{9}\right)$ which can be represented by powers of $\alpha$, where $\alpha$ is the root of irreducible polynomial $p(x)=x^{9}+x^{4}+1$ over $\mathbb{Z}_{2}$. Let $G F^{*}\left(2^{9}\right)$ denote the elements of $G F\left(2^{9}\right)$ which are of the form of even powers of $\alpha$. In the first step, we construct a $16 \times 16$ matrix with the elements of $G F^{*}\left(2^{9}\right)$ in a specific order, determined by the coset diagram. Next, we consider $h: G F^{*}\left(2^{9}\right) \longrightarrow G F\left(2^{8}\right)$ defined by $h\left(\alpha^{2 n}\right)=\omega^{n}$ to destroy the structure of $G F\left(2^{8}\right)$. In the last step, we apply a bijective map $g$ on each element of the matrix to evolve proposed S-box. The ability of the proposed S-box is examined by different available algebraic and statistical analyses. The results are then compared with the familiar S-boxes. We get encouraging statistics of the proposed box after comparison.

\section{Introduction}

In secure communication, the role of the nonlinear component for block ciphers (substitution box) is of significant importance. The concept of substitution box was given by Shannon in 1949 [1]. In order to create confusion during the process of enciphering the digital data, substitution box plays a central role [2]. If the S-box is not good, it means one has to compromise on the quality of encryption. The strength of the S-box affirms the capability of block ciphers. Several attempts have been made to increase the quality of the S-box. In order to assess the properties of wellknown S-boxes, the cryptographers have drawn attention to the literature. Different techniques have been developed to inspect the statistical and algebraic structure of S-boxes. These analyses include linear approximation probability (LP) method, bit independence criterion (BIC), majority logic criterion (MLC), strict avalanche criterion (SAC), nonlinearity method, and differential approximation probability (DP) method.

In this paper, we establish a novel technique to construct substitution boxes by coset diagrams and bijective maps.

\section{Coset Diagrams for Modular Group}

The modular group, denoted by $P S L(2, \mathbb{Z})$, has a finite presentation $\left\langle x, y: x^{2}=y^{3}=1\right\rangle$, where $x$ and $y$ are linear fractional transformations which map $s$ to $-1 / s$ and $s-1 / s$, respectively. Coset diagrams ([3-7]) are the graphical representation of the action of $\operatorname{PSL}(2, \mathbb{Z})$ on $G F\left(p^{n}\right) \cup\{\infty\}$, where $p$ is a prime. Since the order of $y$ is three, its three cycles are represented by triangles. The vertices of the triangles, which are elements of $G F\left(p^{n}\right) \cup\{\infty\}$, are permuted anticlockwise by $y$. Any two of vertices of the triangles are joined by an edge which 


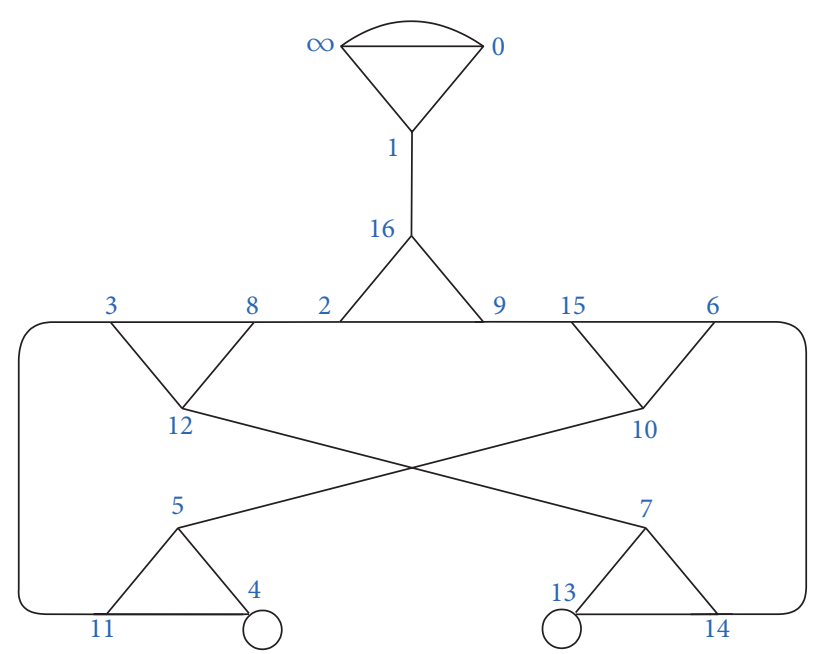

FIGURE 1: The coset diagram for the action of modular group on $G F(17) \cup\{\infty\}$.

represents $x$. The heavy dots are used to denote fixed points of $x$ and $y$, if they exist.
Consider the action of modular group on $G F(17) \cup\{\infty\}$ (Figure 1). The permutation representations of $x$ and $y$ can be calculated by $x: s \rightarrow-1 / s$ and $y: s \rightarrow s-1 / s$.

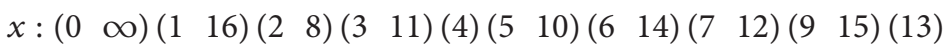

$$
\begin{aligned}
& y:\left(\begin{array}{lllll}
0 & \infty & 1
\end{array}\right)\left(\begin{array}{lllll}
3 & 12 & 8
\end{array}\right)\left(\begin{array}{lllll}
2 & 9 & 16
\end{array}\right)\left(\begin{array}{lllll}
15 & 10 & 6
\end{array}\right)\left(\begin{array}{lllll}
11 & 4 & 5
\end{array}\right)\left(\begin{array}{llll}
13 & 14 & 7
\end{array}\right) .
\end{aligned}
$$

The action of $\operatorname{PSL}(2, \mathbb{Z})$ is not possible on $G F\left(p^{n}\right)$, because image of 0 under $x$ does not belong to $G F\left(p^{n}\right)$. Therefore, we choose $G F\left(p^{n}\right) \cup\{\infty\}$ for the action of $P S L(2, \mathbb{Z})$ instead of $G F\left(p^{n}\right)$.

\section{Coset Diagram Used in the Construction of Proposed Substitution Box}

Consider a primitive irreducible polynomial $p(x)=x^{9}+x^{4}+1$ over $\mathbb{Z}_{2}$; then $G F\left(2^{9}\right)=\mathbb{Z}_{2}[x] /\left\langle x^{9}+x^{4}+1\right\rangle$. The element of
$G F\left(2^{9}\right)$ (see Table 1) can be represented by some power of $\alpha$, where $\alpha$ is the root of $p(x)$. Consider the action of $\operatorname{PSL}(2, \mathbb{Z})$ on $G F\left(2^{9}\right) \cup\{\infty\}$. Then the permutation representations of $x$ and $y$ can be calculated by $(s) x=-1 / s$ and $(s) y=(s-1) / s$, respectively. So

$$
\begin{aligned}
& x:\left(\begin{array}{ll}
\alpha^{1} & \alpha^{510}
\end{array}\right)\left(\begin{array}{ll}
\alpha^{129} & \alpha^{382}
\end{array}\right)\left(\begin{array}{ll}
\alpha^{381} & \alpha^{130}
\end{array}\right)\left(\begin{array}{ll}
\alpha^{2} & \alpha^{509}
\end{array}\right)\left(\begin{array}{ll}
\alpha^{258} & \alpha^{253}
\end{array}\right)\left(\begin{array}{ll}
\alpha^{251} & \alpha^{260}
\end{array}\right)\left(\begin{array}{ll}
\alpha^{3} & \alpha^{508}
\end{array}\right) \\
& \left(\begin{array}{ll}
\alpha^{417} & \alpha^{94}
\end{array}\right)\left(\begin{array}{ll}
\alpha^{91} & \alpha^{420}
\end{array}\right)\left(\begin{array}{ll}
\alpha^{2} & \alpha^{509}
\end{array}\right)\left(\begin{array}{ll}
\alpha^{258} & \alpha^{253}
\end{array}\right)\left(\begin{array}{ll}
\alpha^{251} & \alpha^{260}
\end{array}\right)\left(\begin{array}{ll}
\alpha^{4} & \alpha^{507}
\end{array}\right)\left(\begin{array}{ll}
\alpha^{5} & \alpha^{506}
\end{array}\right) \\
& \left(\begin{array}{ll}
\alpha^{502} & \alpha^{9}
\end{array}\right)\left(\begin{array}{ll}
\alpha^{6} & \alpha^{505}
\end{array}\right)\left(\alpha^{323} \alpha^{188}\right)\left(\begin{array}{ll}
\alpha^{182} & \alpha^{329}
\end{array}\right)\left(\begin{array}{ll}
\alpha^{7} & \alpha^{504}
\end{array}\right)\left(\begin{array}{ll}
\alpha^{60} & \alpha^{451}
\end{array}\right)\left(\begin{array}{ll}
\alpha^{444} & \alpha^{67}
\end{array}\right) \\
& \left(\begin{array}{ll}
\alpha^{8} & \alpha^{503}
\end{array}\right)\left(\begin{array}{ll}
\alpha^{10} & \alpha^{501}
\end{array}\right)\left(\begin{array}{ll}
\alpha^{493} & \alpha^{18}
\end{array}\right)\left(\begin{array}{ll}
\alpha^{11} & \alpha^{500}
\end{array}\right)\left(\begin{array}{ll}
\alpha^{459} & \alpha^{52}
\end{array}\right)\left(\begin{array}{ll}
\alpha^{41} & \alpha^{470}
\end{array}\right)\left(\begin{array}{ll}
\alpha^{12} & \alpha^{499}
\end{array}\right) \\
& \left(\alpha^{135} \alpha^{376}\right)\left(\alpha^{364} \alpha^{147}\right)\left(\alpha^{13} \alpha^{498}\right)\left(\alpha^{125} \alpha^{386}\right)\left(\alpha^{373} \alpha^{138}\right)\left(\alpha^{14} \alpha^{497}\right)\left(\begin{array}{ll}
\alpha^{120} & \alpha^{391}
\end{array}\right) \\
& \left(\alpha^{377} \alpha^{134}\right)\left(\alpha^{15} \alpha^{496}\right)\left(\alpha^{111} \alpha^{400}\right)\left(\alpha^{385} \alpha^{126}\right)\left(\alpha^{16} \alpha^{495}\right)\left(\alpha^{20} \alpha^{491}\right)\left(\alpha^{475} \alpha^{36}\right)
\end{aligned}
$$


TABLE 1: Representation of the elements of $G F\left(2^{9}\right)$.

\begin{tabular}{|c|c|c|c|c|c|c|c|}
\hline Binary values & $G F\left(2^{9}\right)$ & Binary values & $G F\left(2^{9}\right)$ & Binary values & $G F\left(2^{9}\right)$ & Binary values & $G F\left(2^{9}\right)$ \\
\hline 000000000 & 0 & 000000001 & 1 & 000000010 & $\alpha^{1}$ & 000000100 & $\alpha^{2}$ \\
\hline 000001000 & $\alpha^{3}$ & 000010000 & $\alpha^{4}$ & 000100000 & $\alpha^{5}$ & 001000000 & $\alpha^{6}$ \\
\hline 010000000 & $\alpha^{7}$ & 100000000 & $\alpha^{8}$ & 000010001 & $\alpha^{9}$ & 000100010 & $\alpha^{10}$ \\
\hline 001000100 & $\alpha^{11}$ & 010001000 & $\alpha^{12}$ & 100010000 & $\alpha^{13}$ & 000110001 & $\alpha^{14}$ \\
\hline 001100010 & $\alpha^{15}$ & 011000100 & $\alpha^{16}$ & 110001000 & $\alpha^{17}$ & 100000001 & $\alpha^{18}$ \\
\hline 000010011 & $\alpha^{19}$ & 000100110 & $\alpha^{20}$ & 001001100 & $\alpha^{21}$ & 010011000 & $\alpha^{22}$ \\
\hline 100110000 & $\alpha^{23}$ & 001110001 & $\alpha^{24}$ & 011100010 & $\alpha^{25}$ & 111000100 & $\alpha^{26}$ \\
\hline 110011001 & $\alpha^{27}$ & 100100011 & $\alpha^{28}$ & 001010111 & $\alpha^{29}$ & 010101110 & $\alpha^{30}$ \\
\hline 101011100 & $\alpha^{31}$ & 010101001 & $\alpha^{32}$ & 101010010 & $\alpha^{33}$ & 010110101 & $\alpha^{34}$ \\
\hline 101101010 & $\alpha^{35}$ & 011000101 & $\alpha^{36}$ & 110001010 & $\alpha^{37}$ & 100000101 & $\alpha^{38}$ \\
\hline 000011011 & $\alpha^{39}$ & 000110110 & $\alpha^{40}$ & 001101100 & $\alpha^{41}$ & 011011000 & $\alpha^{42}$ \\
\hline 110110000 & $\alpha^{43}$ & 101110001 & $\alpha^{44}$ & 011110011 & $\alpha^{45}$ & 111100110 & $\alpha^{46}$ \\
\hline 111011101 & $\alpha^{47}$ & 110101011 & $\alpha^{48}$ & 101000111 & $\alpha^{49}$ & 010011111 & $\alpha^{50}$ \\
\hline 100111110 & $\alpha^{51}$ & 001101101 & $\alpha^{52}$ & 011011010 & $\alpha^{53}$ & 110110100 & $\alpha^{54}$ \\
\hline 101111001 & $\alpha^{55}$ & 011100011 & $\alpha^{56}$ & 111000110 & $\alpha^{57}$ & 110011101 & $\alpha^{58}$ \\
\hline 100101011 & $\alpha^{59}$ & 001000111 & $\alpha^{60}$ & 010001110 & $\alpha^{61}$ & 100011100 & $\alpha^{62}$ \\
\hline 000101001 & $\alpha^{63}$ & 001010010 & $\alpha^{64}$ & 010100100 & $\alpha^{65}$ & 101001000 & $\alpha^{66}$ \\
\hline 010000001 & $\alpha^{67}$ & 100000010 & $\alpha^{68}$ & 000010101 & $\alpha^{69}$ & 000101010 & $\alpha^{70}$ \\
\hline 001010100 & $\alpha^{71}$ & 010101000 & $\alpha^{72}$ & 101010000 & $\alpha^{73}$ & 010110001 & $\alpha^{74}$ \\
\hline 101100010 & $\alpha^{75}$ & 011010101 & $\alpha^{76}$ & 110101010 & $\alpha^{77}$ & 101000101 & $\alpha^{78}$ \\
\hline 010011011 & $\alpha^{79}$ & 100110110 & $\alpha^{80}$ & 001111101 & $\alpha^{81}$ & 011111010 & $\alpha^{82}$ \\
\hline 111110100 & $\alpha^{83}$ & 111111001 & $\alpha^{84}$ & 111100011 & $\alpha^{85}$ & 111010111 & $\alpha^{86}$ \\
\hline 110111111 & $\alpha^{87}$ & 101101111 & $\alpha^{88}$ & 011001111 & $\alpha^{89}$ & 110011110 & $\alpha^{90}$ \\
\hline 100101101 & $\alpha^{91}$ & 001001011 & $\alpha^{92}$ & 010010110 & $\alpha^{93}$ & 100101100 & $\alpha^{94}$ \\
\hline 001001001 & $\alpha^{95}$ & 010010010 & $\alpha^{96}$ & 100100100 & $\alpha^{97}$ & 001011001 & $\alpha^{98}$ \\
\hline 010110010 & $\alpha^{99}$ & 101100100 & $\alpha^{100}$ & 011011001 & $\alpha^{101}$ & 110110010 & $\alpha^{102}$ \\
\hline 101110101 & $\alpha^{103}$ & 011111011 & $\alpha^{104}$ & 111110110 & $\alpha^{105}$ & 111111101 & $\alpha^{106}$ \\
\hline 111101011 & $\alpha^{107}$ & 111000111 & $\alpha^{108}$ & 110011111 & $\alpha^{109}$ & 100101111 & $\alpha^{110}$ \\
\hline 001001111 & $\alpha^{111}$ & 010011110 & $\alpha^{112}$ & 100111100 & $\alpha^{113}$ & 001101001 & $\alpha^{114}$ \\
\hline 011010010 & $\alpha^{115}$ & 110100100 & $\alpha^{116}$ & 101011001 & $\alpha^{117}$ & 010100011 & $\alpha^{118}$ \\
\hline 101000110 & $\alpha^{119}$ & 010011101 & $\alpha^{120}$ & 100111010 & $\alpha^{121}$ & 001100101 & $\alpha^{122}$ \\
\hline 011001010 & $\alpha^{123}$ & 110010100 & $\alpha^{124}$ & 100111001 & $\alpha^{125}$ & 001100011 & $\alpha^{126}$ \\
\hline 011000110 & $\alpha^{127}$ & 110001100 & $\alpha^{128}$ & 100001001 & $\alpha^{129}$ & 000000011 & $\alpha^{130}$ \\
\hline 000000110 & $\alpha^{131}$ & 000001100 & $\alpha^{132}$ & 000011000 & $\alpha^{133}$ & 000110000 & $\alpha^{134}$ \\
\hline 001100000 & $\alpha^{135}$ & 011000000 & $\alpha^{136}$ & 110000000 & $\alpha^{137}$ & 100010001 & $\alpha^{138}$ \\
\hline 000110011 & $\alpha^{139}$ & 001100110 & $\alpha^{140}$ & 011001100 & $\alpha^{141}$ & 110011000 & $\alpha^{142}$ \\
\hline 100100001 & $\alpha^{143}$ & 001010011 & $\alpha^{144}$ & 010100110 & $\alpha^{145}$ & 101001100 & $\alpha^{146}$ \\
\hline 010001001 & $\alpha^{147}$ & 100010010 & $\alpha^{148}$ & 000110101 & $\alpha^{149}$ & 001101010 & $\alpha^{150}$ \\
\hline 011010100 & $\alpha^{151}$ & 110101000 & $\alpha^{152}$ & 101000001 & $\alpha^{153}$ & 010010011 & $\alpha^{154}$ \\
\hline 100100110 & $\alpha^{155}$ & 001011101 & $\alpha^{156}$ & 010111010 & $\alpha^{157}$ & 101110100 & $\alpha^{158}$ \\
\hline 011111001 & $\alpha^{159}$ & 111110010 & $\alpha^{160}$ & 111110101 & $\alpha^{161}$ & 111111011 & $\alpha^{162}$ \\
\hline 111100111 & $\alpha^{163}$ & 111011111 & $\alpha^{164}$ & 110101111 & $\alpha^{165}$ & 101001111 & $\alpha^{166}$ \\
\hline 010001111 & $\alpha^{167}$ & 100011110 & $\alpha^{168}$ & 000101101 & $\alpha^{169}$ & 001011010 & $\alpha^{170}$ \\
\hline 010110100 & $\alpha^{171}$ & 101101000 & $\alpha^{172}$ & 011000001 & $\alpha^{173}$ & 110000010 & $\alpha^{174}$ \\
\hline 100010101 & $\alpha^{175}$ & 000111011 & $\alpha^{176}$ & 001110110 & $\alpha^{177}$ & 011101100 & $\alpha^{178}$ \\
\hline 111011000 & $\alpha^{179}$ & 110100001 & $\alpha^{180}$ & 101010011 & $\alpha^{181}$ & 010110111 & $\alpha^{182}$ \\
\hline 101101110 & $\alpha^{183}$ & 011001101 & $\alpha^{184}$ & 110011010 & $\alpha^{185}$ & 100100101 & $\alpha^{186}$ \\
\hline 001011011 & $\alpha^{187}$ & 010110110 & $\alpha^{188}$ & 101101100 & $\alpha^{189}$ & 011001001 & $\alpha^{190}$ \\
\hline 110010010 & $\alpha^{191}$ & 100110101 & $\alpha^{192}$ & 001111011 & $\alpha^{193}$ & 011110110 & $\alpha^{194}$ \\
\hline 111101100 & $\alpha^{195}$ & 111001001 & $\alpha^{196}$ & 110000011 & $\alpha^{197}$ & 100010111 & $\alpha^{198}$ \\
\hline 000111111 & $\alpha^{199}$ & 0011111110 & $\alpha^{200}$ & 011111100 & $\alpha^{201}$ & 1111111000 & $\alpha^{202}$ \\
\hline 111100001 & $\alpha^{203}$ & 111010011 & $\alpha^{204}$ & 110110111 & $\alpha^{205}$ & 101111111 & $\alpha^{206}$ \\
\hline
\end{tabular}


TABle 1: Continued.

\begin{tabular}{|c|c|c|c|c|c|c|c|}
\hline Binary values & $G F\left(2^{9}\right)$ & Binary values & $G F\left(2^{9}\right)$ & Binary values & $G F\left(2^{9}\right)$ & Binary values & $G F\left(2^{9}\right)$ \\
\hline 011101111 & $\alpha^{207}$ & 111011110 & $\alpha^{208}$ & 110101101 & $\alpha^{209}$ & 101001011 & $\alpha^{210}$ \\
\hline 010000111 & $\alpha^{211}$ & 100001110 & $\alpha^{212}$ & 000001101 & $\alpha^{213}$ & 000011010 & $\alpha^{214}$ \\
\hline 000110100 & $\alpha^{215}$ & 001101000 & $\alpha^{216}$ & 011010000 & $\alpha^{217}$ & 110100000 & $\alpha^{218}$ \\
\hline 101010001 & $\alpha^{219}$ & 010110011 & $\alpha^{220}$ & 101100110 & $\alpha^{221}$ & 011011101 & $\alpha^{222}$ \\
\hline 110111010 & $\alpha^{223}$ & 101100101 & $\alpha^{224}$ & 011011011 & $\alpha^{225}$ & 110110110 & $\alpha^{226}$ \\
\hline 101111101 & $\alpha^{227}$ & 011101011 & $\alpha^{228}$ & 111010110 & $\alpha^{229}$ & 110111101 & $\alpha^{230}$ \\
\hline 101101011 & $\alpha^{231}$ & 011000111 & $\alpha^{232}$ & 110001110 & $\alpha^{233}$ & 100001101 & $\alpha^{234}$ \\
\hline 000001011 & $\alpha^{235}$ & 000010110 & $\alpha^{236}$ & 000101100 & $\alpha^{237}$ & 001011000 & $\alpha^{238}$ \\
\hline 010110000 & $\alpha^{239}$ & 101100000 & $\alpha^{240}$ & 011010001 & $\alpha^{241}$ & 110100010 & $\alpha^{242}$ \\
\hline 101010101 & $\alpha^{243}$ & 010111011 & $\alpha^{244}$ & 101110110 & $\alpha^{245}$ & 011111101 & $\alpha^{246}$ \\
\hline 111111010 & $\alpha^{247}$ & 111100101 & $\alpha^{248}$ & 111011011 & $\alpha^{249}$ & 110100111 & $\alpha^{250}$ \\
\hline 101011111 & $\alpha^{251}$ & 010101111 & $\alpha^{252}$ & 101011110 & $\alpha^{253}$ & 010101101 & $\alpha^{254}$ \\
\hline 101011010 & $\alpha^{255}$ & 010100101 & $\alpha^{256}$ & 101001010 & $\alpha^{257}$ & 010000101 & $\alpha^{258}$ \\
\hline 100001010 & $\alpha^{259}$ & 000000101 & $\alpha^{260}$ & 000001010 & $\alpha^{261}$ & 000010100 & $\alpha^{262}$ \\
\hline 000101000 & $\alpha^{263}$ & 001010000 & $\alpha^{264}$ & 010100000 & $\alpha^{265}$ & 101000000 & $\alpha^{266}$ \\
\hline 010010001 & $\alpha^{267}$ & 100100010 & $\alpha^{268}$ & 001010101 & $\alpha^{269}$ & 010101010 & $\alpha^{270}$ \\
\hline 101010100 & $\alpha^{271}$ & 010111001 & $\alpha^{272}$ & 101110010 & $\alpha^{273}$ & 011110101 & $\alpha^{274}$ \\
\hline 111101010 & $\alpha^{275}$ & 111000101 & $\alpha^{276}$ & 110011011 & $\alpha^{277}$ & 100100111 & $\alpha^{278}$ \\
\hline 001011111 & $\alpha^{279}$ & 010111110 & $\alpha^{280}$ & 101111100 & $\alpha^{281}$ & 011101001 & $\alpha^{282}$ \\
\hline 111010010 & $\alpha^{283}$ & 110110101 & $\alpha^{284}$ & 101111011 & $\alpha^{285}$ & 011100111 & $\alpha^{286}$ \\
\hline 111001110 & $\alpha^{287}$ & 110001101 & $\alpha^{288}$ & 100001011 & $\alpha^{289}$ & 000000111 & $\alpha^{290}$ \\
\hline 000001110 & $\alpha^{291}$ & 000011100 & $\alpha^{292}$ & 000111000 & $\alpha^{293}$ & 001110000 & $\alpha^{294}$ \\
\hline 011100000 & $\alpha^{295}$ & 111000000 & $\alpha^{296}$ & 110010001 & $\alpha^{297}$ & 100110011 & $\alpha^{298}$ \\
\hline 001110111 & $\alpha^{299}$ & 011101110 & $\alpha^{300}$ & 111011100 & $\alpha^{301}$ & 110101001 & $\alpha^{302}$ \\
\hline 101000011 & $\alpha^{303}$ & 010010111 & $\alpha^{304}$ & 100101110 & $\alpha^{305}$ & 001001101 & $\alpha^{306}$ \\
\hline 010011010 & $\alpha^{307}$ & 100110100 & $\alpha^{308}$ & 001111001 & $\alpha^{309}$ & 011110010 & $\alpha^{310}$ \\
\hline 111100100 & $\alpha^{311}$ & 111011001 & $\alpha^{312}$ & 110100011 & $\alpha^{313}$ & 101010111 & $\alpha^{314}$ \\
\hline 010111111 & $\alpha^{315}$ & 101111110 & $\alpha^{316}$ & 011101101 & $\alpha^{317}$ & 111011010 & $\alpha^{318}$ \\
\hline 110100101 & $\alpha^{319}$ & 101011011 & $\alpha^{320}$ & 010100111 & $\alpha^{321}$ & 101001110 & $\alpha^{322}$ \\
\hline 010001101 & $\alpha^{323}$ & 100011010 & $\alpha^{324}$ & 000100101 & $\alpha^{325}$ & 001001010 & $\alpha^{326}$ \\
\hline 010010100 & $\alpha^{327}$ & 100101000 & $\alpha^{328}$ & 001000001 & $\alpha^{329}$ & 010000010 & $\alpha^{330}$ \\
\hline 100000100 & $\alpha^{331}$ & 000011001 & $\alpha^{332}$ & 000110010 & $\alpha^{333}$ & 001100100 & $\alpha^{334}$ \\
\hline 011001000 & $\alpha^{335}$ & 110010000 & $\alpha^{336}$ & 100110001 & $\alpha^{337}$ & 001110011 & $\alpha^{338}$ \\
\hline 011100110 & $\alpha^{339}$ & 111001100 & $\alpha^{340}$ & 110001001 & $\alpha^{341}$ & 100000011 & $\alpha^{342}$ \\
\hline 000010111 & $\alpha^{343}$ & 000101110 & $\alpha^{344}$ & 001011100 & $\alpha^{345}$ & 010111000 & $\alpha^{346}$ \\
\hline 101110000 & $\alpha^{347}$ & 011110001 & $\alpha^{348}$ & 111100010 & $\alpha^{349}$ & 111010101 & $\alpha^{350}$ \\
\hline 110111011 & $\alpha^{351}$ & 101100111 & $\alpha^{352}$ & 011011111 & $\alpha^{353}$ & 110111110 & $\alpha^{354}$ \\
\hline 101101101 & $\alpha^{355}$ & 011001011 & $\alpha^{356}$ & 110010110 & $\alpha^{357}$ & 100111101 & $\alpha^{358}$ \\
\hline 001101011 & $\alpha^{359}$ & 011010110 & $\alpha^{360}$ & 110101100 & $\alpha^{361}$ & 101001001 & $\alpha^{362}$ \\
\hline 010000011 & $\alpha^{363}$ & 100000110 & $\alpha^{364}$ & 000011101 & $\alpha^{365}$ & 000111010 & $\alpha^{366}$ \\
\hline 001110100 & $\alpha^{367}$ & 011101000 & $\alpha^{368}$ & 111010000 & $\alpha^{369}$ & 110110001 & $\alpha^{370}$ \\
\hline 101110011 & $\alpha^{371}$ & 011110111 & $\alpha^{372}$ & 111101110 & $\alpha^{373}$ & 111001101 & $\alpha^{374}$ \\
\hline 110001011 & $\alpha^{375}$ & 100000111 & $\alpha^{376}$ & 000011111 & $\alpha^{377}$ & 000111110 & $\alpha^{378}$ \\
\hline 001111100 & $\alpha^{379}$ & 011111000 & $\alpha^{380}$ & 111110000 & $\alpha^{381}$ & 111110001 & $\alpha^{382}$ \\
\hline 111110011 & $\alpha^{383}$ & 111110111 & $\alpha^{384}$ & 111111111 & $\alpha^{385}$ & 111101111 & $\alpha^{386}$ \\
\hline 111001111 & $\alpha^{387}$ & 110001111 & $\alpha^{388}$ & 100001111 & $\alpha^{389}$ & 000001111 & $\alpha^{390}$ \\
\hline 000011110 & $\alpha^{391}$ & 000111100 & $\alpha^{392}$ & 001111000 & $\alpha^{393}$ & 011110000 & $\alpha^{394}$ \\
\hline 111100000 & $\alpha^{395}$ & 111010001 & $\alpha^{396}$ & 110110011 & $\alpha^{397}$ & 101110111 & $\alpha^{398}$ \\
\hline 011111111 & $\alpha^{399}$ & 111111110 & $\alpha^{400}$ & 111101101 & $\alpha^{401}$ & 111001011 & $\alpha^{402}$ \\
\hline 110000111 & $\alpha^{403}$ & 100011111 & $\alpha^{404}$ & 000101111 & $\alpha^{405}$ & 001011110 & $\alpha^{406}$ \\
\hline 010111100 & $\alpha^{407}$ & 101111000 & $\alpha^{408}$ & 011100001 & $\alpha^{409}$ & 111000010 & $\alpha^{410}$ \\
\hline 110010101 & $\alpha^{411}$ & 100111011 & $\alpha^{412}$ & 001100111 & $\alpha^{413}$ & 011001110 & $\alpha^{414}$ \\
\hline
\end{tabular}


TABle 1: Continued.

\begin{tabular}{|c|c|c|c|c|c|c|c|}
\hline Binary values & $G F\left(2^{9}\right)$ & Binary values & $G F\left(2^{9}\right)$ & Binary values & $G F\left(2^{9}\right)$ & Binary values & $G F\left(2^{9}\right)$ \\
\hline 110011100 & $\alpha^{415}$ & 100101001 & $\alpha^{416}$ & 001000011 & $\alpha^{417}$ & 010000110 & $\alpha^{418}$ \\
\hline 100001100 & $\alpha^{419}$ & 000001001 & $\alpha^{420}$ & 000010010 & $\alpha^{421}$ & 000100100 & $\alpha^{422}$ \\
\hline 001001000 & $\alpha^{423}$ & 010010000 & $\alpha^{424}$ & 100100000 & $\alpha^{425}$ & 001010001 & $\alpha^{426}$ \\
\hline 010100010 & $\alpha^{427}$ & 101000100 & $\alpha^{428}$ & 010011001 & $\alpha^{429}$ & 100110010 & $\alpha^{430}$ \\
\hline 001110101 & $\alpha^{431}$ & 011101010 & $\alpha^{432}$ & 111010100 & $\alpha^{433}$ & 110111001 & $\alpha^{434}$ \\
\hline 101100011 & $\alpha^{435}$ & 011010111 & $\alpha^{436}$ & 110101110 & $\alpha^{437}$ & 101001101 & $\alpha^{438}$ \\
\hline 010001011 & $\alpha^{439}$ & 100010110 & $\alpha^{440}$ & 000111101 & $\alpha^{441}$ & 001111010 & $\alpha^{442}$ \\
\hline 011110100 & $\alpha^{443}$ & 111101000 & $\alpha^{444}$ & 111000001 & $\alpha^{445}$ & 110010011 & $\alpha^{446}$ \\
\hline 100110111 & $\alpha^{447}$ & 001111111 & $\alpha^{448}$ & 011111110 & $\alpha^{449}$ & 111111100 & $\alpha^{450}$ \\
\hline 111101001 & $\alpha^{451}$ & 111000011 & $\alpha^{452}$ & 110010111 & $\alpha^{453}$ & 100111111 & $\alpha^{454}$ \\
\hline 001101111 & $\alpha^{455}$ & 011011110 & $\alpha^{456}$ & 110111100 & $\alpha^{457}$ & 101101001 & $\alpha^{458}$ \\
\hline 011000011 & $\alpha^{459}$ & 110000110 & $\alpha^{460}$ & 100011101 & $\alpha^{461}$ & 000101011 & $\alpha^{462}$ \\
\hline 001010110 & $\alpha^{463}$ & 010101100 & $\alpha^{464}$ & 101011000 & $\alpha^{465}$ & 010100001 & $\alpha^{466}$ \\
\hline 101000010 & $\alpha^{467}$ & 010010101 & $\alpha^{468}$ & 100101010 & $\alpha^{469}$ & 001000101 & $\alpha^{470}$ \\
\hline 010001010 & $\alpha^{471}$ & 100010100 & $\alpha^{472}$ & 000111001 & $\alpha^{473}$ & 001110010 & $\alpha^{474}$ \\
\hline 011100100 & $\alpha^{475}$ & 111001000 & $\alpha^{476}$ & 110000001 & $\alpha^{477}$ & 100010011 & $\alpha^{478}$ \\
\hline 000110111 & $\alpha^{479}$ & 001101110 & $\alpha^{480}$ & 011011100 & $\alpha^{481}$ & 110111000 & $\alpha^{482}$ \\
\hline 101100001 & $\alpha^{483}$ & 011010011 & $\alpha^{484}$ & 110100110 & $\alpha^{485}$ & 101011101 & $\alpha^{486}$ \\
\hline 010101011 & $\alpha^{487}$ & 101010110 & $\alpha^{488}$ & 010111101 & $\alpha^{489}$ & 101111010 & $\alpha^{490}$ \\
\hline 011100101 & $\alpha^{491}$ & 111001010 & $\alpha^{492}$ & 110000101 & $\alpha^{493}$ & 100011011 & $\alpha^{494}$ \\
\hline 000100111 & $\alpha^{495}$ & 001001110 & $\alpha^{496}$ & 010011100 & $\alpha^{497}$ & 100111000 & $\alpha^{498}$ \\
\hline 001100001 & $\alpha^{499}$ & 011000010 & $\alpha^{500}$ & 110000100 & $\alpha^{501}$ & 100011001 & $\alpha^{502}$ \\
\hline 000100011 & $\alpha^{503}$ & 001000110 & $\alpha^{504}$ & 010001100 & $\alpha^{505}$ & 100011000 & $\alpha^{506}$ \\
\hline 000100001 & $\alpha^{507}$ & 001000010 & $\alpha^{508}$ & 010000100 & $\alpha^{509}$ & 100001000 & $\alpha^{510}$ \\
\hline
\end{tabular}

$$
\begin{aligned}
& \left(\begin{array}{ll}
\alpha^{17} & \alpha^{494}
\end{array}\right)\left(\alpha^{324} \alpha^{187}\right)\left(\begin{array}{cc}
\alpha^{170} & \alpha^{341}
\end{array}\right)\left(\begin{array}{ll}
\alpha^{19} & \alpha^{492}
\end{array}\right)\left(\begin{array}{cc}
\alpha^{402} & \alpha^{109}
\end{array}\right)\left(\begin{array}{ll}
\alpha^{90} & \alpha^{421}
\end{array}\right)\left(\begin{array}{ll}
\alpha^{21} & \alpha^{490}
\end{array}\right) \\
& \left(\begin{array}{ll}
\alpha^{285} & \alpha^{226}
\end{array}\right)\left(\begin{array}{ll}
\alpha^{306} & \alpha^{205}
\end{array}\right)\left(\begin{array}{ll}
\alpha^{22} & \alpha^{489}
\end{array}\right)\left(\alpha^{407} \alpha^{104}\right)\left(\begin{array}{ll}
\alpha^{82} & \alpha^{429}
\end{array}\right)\left(\begin{array}{ll}
\alpha^{23} & \alpha^{488}
\end{array}\right)\left(\begin{array}{lll}
\alpha^{314} & \alpha^{197}
\end{array}\right) \\
& \left(\begin{array}{ll}
\alpha^{174} & \alpha^{337}
\end{array}\right)\left(\begin{array}{ll}
\alpha^{24} & \alpha^{487}
\end{array}\right)\left(\begin{array}{ll}
\alpha^{270} & \alpha^{241}
\end{array}\right)\left(\begin{array}{ll}
\alpha^{217} & \alpha^{294}
\end{array}\right)\left(\begin{array}{ll}
\alpha^{25} & \alpha^{486}
\end{array}\right)\left(\alpha^{31} \alpha^{480}\right)\left(\begin{array}{ll}
\alpha^{455} & \alpha^{56}
\end{array}\right) \\
& \left(\begin{array}{ll}
\alpha^{26} & \alpha^{485}
\end{array}\right)\left(\begin{array}{ll}
\alpha^{250} & \alpha^{261}
\end{array}\right)\left(\begin{array}{ll}
\alpha^{235} & \alpha^{276}
\end{array}\right)\left(\begin{array}{ll}
\alpha^{27} & \alpha^{484}
\end{array}\right)\left(\begin{array}{ll}
\alpha^{115} & \alpha^{396}
\end{array}\right)\left(\begin{array}{ll}
\alpha^{369} & \alpha^{142}
\end{array}\right)\left(\begin{array}{ll}
\alpha^{455} & \alpha^{56}
\end{array}\right) \\
& \left(\begin{array}{ll}
\alpha^{28} & \alpha^{483}
\end{array}\right)\left(\begin{array}{ll}
\alpha^{240} & \alpha^{271}
\end{array}\right)\left(\begin{array}{ll}
\alpha^{243} & \alpha^{268}
\end{array}\right)\left(\begin{array}{ll}
\alpha^{29} & \alpha^{482}
\end{array}\right)\left(\begin{array}{ll}
\alpha^{434} & \alpha^{77}
\end{array}\right)\left(\begin{array}{ll}
\alpha^{48} & \alpha^{463}
\end{array}\right)\left(\begin{array}{ll}
\alpha^{30} & \alpha^{481}
\end{array}\right) \\
& \left(\begin{array}{ll}
\alpha^{222} & \alpha^{289}
\end{array}\right)\left(\begin{array}{ll}
\alpha^{259} & \alpha^{252}
\end{array}\right)\left(\begin{array}{ll}
\alpha^{32} & \alpha^{479}
\end{array}\right)\left(\begin{array}{ll}
\alpha^{40} & \alpha^{471}
\end{array}\right)\left(\begin{array}{ll}
\alpha^{439} & \alpha^{72}
\end{array}\right)\left(\begin{array}{ll}
\alpha^{33} & \alpha^{478}
\end{array}\right)\left(\begin{array}{ll}
\alpha^{148} & \alpha^{363}
\end{array}\right) \\
& \left(\begin{array}{ll}
\alpha^{330} & \alpha^{181}
\end{array}\right)\left(\alpha^{34} \alpha^{477}\right)\left(\alpha^{137} \alpha^{374}\right)\left(\begin{array}{ll}
\alpha^{340} & \alpha^{171}
\end{array}\right)\left(\begin{array}{ll}
\alpha^{35} & \alpha^{476}
\end{array}\right)\left(\begin{array}{ll}
\alpha^{196} & \alpha^{315}
\end{array}\right)\left(\begin{array}{ll}
\alpha^{280} & \alpha^{231}
\end{array}\right)
\end{aligned}
$$

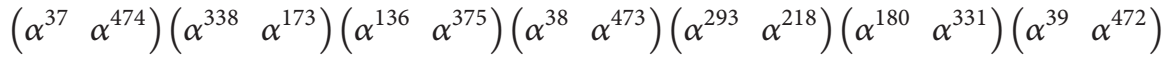

$$
\begin{aligned}
& \left(\begin{array}{ll}
\alpha^{175} & \alpha^{336}
\end{array}\right)\left(\begin{array}{ll}
\alpha^{297} & \alpha^{214}
\end{array}\right)\left(\begin{array}{ll}
\alpha^{42} & \alpha^{469}
\end{array}\right)\left(\begin{array}{ll}
\alpha^{59} & \alpha^{452}
\end{array}\right)\left(\begin{array}{ll}
\alpha^{410} & \alpha^{101}
\end{array}\right)\left(\begin{array}{ll}
\alpha^{43} & \alpha^{468}
\end{array}\right)\left(\alpha^{327} \alpha^{184}\right) \\
& \left(\begin{array}{ll}
\alpha^{141} & \alpha^{370}
\end{array}\right)\left(\begin{array}{ll}
\alpha^{44} & \alpha^{467}
\end{array}\right)\left(\begin{array}{ll}
\alpha^{303} & \alpha^{208}
\end{array}\right)\left(\begin{array}{ll}
\alpha^{164} & \alpha^{347}
\end{array}\right)\left(\begin{array}{ll}
\alpha^{45} & \alpha^{466}
\end{array}\right)\left(\begin{array}{ll}
\alpha^{265} & \alpha^{246}
\end{array}\right)\left(\begin{array}{ll}
\alpha^{201} & \alpha^{310}
\end{array}\right) \\
& \left(\begin{array}{ll}
\alpha^{46} & \alpha^{465}
\end{array}\right)\left(\begin{array}{ll}
\alpha^{117} & \alpha^{394}
\end{array}\right)\left(\begin{array}{ll}
\alpha^{348} & \alpha^{163}
\end{array}\right)\left(\begin{array}{ll}
\alpha^{47} & \alpha^{464}
\end{array}\right)\left(\begin{array}{lll}
\alpha^{254} & \alpha^{257}
\end{array}\right)\left(\begin{array}{ll}
\alpha^{210} & \alpha^{301}
\end{array}\right)\left(\begin{array}{ll}
\alpha^{49} & \alpha^{462}
\end{array}\right) \\
& \left(\begin{array}{ll}
\alpha^{70} & \alpha^{441}
\end{array}\right)\left(\alpha^{392} \alpha^{119}\right)\left(\begin{array}{ll}
\alpha^{50} & \alpha^{461}
\end{array}\right)\left(\begin{array}{ll}
\alpha^{62} & \alpha^{449}
\end{array}\right)\left(\begin{array}{cc}
\alpha^{51} & \alpha^{460}
\end{array}\right)\left(\alpha^{403} \alpha^{108}\right)\left(\alpha^{57} \alpha^{454}\right) \\
& \left(\begin{array}{ll}
\alpha^{53} & \alpha^{458}
\end{array}\right)\left(\alpha^{172} \alpha^{339}\right)\left(\begin{array}{cc}
\alpha^{286} & \alpha^{225}
\end{array}\right)\left(\alpha^{54} \alpha^{457}\right)\left(\alpha^{230} \alpha^{281}\right)\left(\alpha^{227} \alpha^{284}\right)\left(\alpha^{55} \alpha^{456}\right) \\
& \left(\alpha^{353} \alpha^{158}\right)\left(\alpha^{103} \alpha^{408}\right)\left(\begin{array}{ll}
\alpha^{58} & \alpha^{453}
\end{array}\right)\left(\alpha^{357} \alpha^{154}\right)\left(\begin{array}{lll}
\alpha^{96} & \alpha^{415}
\end{array}\right)\left(\alpha^{61} \alpha^{450}\right)\left(\alpha^{106} \alpha^{405}\right)
\end{aligned}
$$




$$
\begin{aligned}
& \left(\begin{array}{ll}
\alpha^{344} & \alpha^{167}
\end{array}\right)\left(\begin{array}{ll}
\alpha^{63} & \alpha^{448}
\end{array}\right)\left(\begin{array}{ll}
\alpha^{200} & \alpha^{311}
\end{array}\right)\left(\begin{array}{ll}
\alpha^{248} & \alpha^{263}
\end{array}\right)\left(\begin{array}{ll}
\alpha^{64} & \alpha^{447}
\end{array}\right)\left(\begin{array}{ll}
\alpha^{80} & \alpha^{431}
\end{array}\right)\left(\begin{array}{ll}
\alpha^{367} & \alpha^{144}
\end{array}\right) \\
& \left(\alpha^{65} \alpha^{446}\right)\left(\alpha^{191} \alpha^{320}\right)\left(\alpha^{255} \alpha^{256}\right)\left(\alpha^{66} \alpha^{445}\right)\left(\alpha^{296} \alpha^{215}\right)\left(\begin{array}{ll}
\alpha^{149} & \alpha^{362}
\end{array}\right)\left(\begin{array}{ll}
\alpha^{68} & \alpha^{443}
\end{array}\right) \\
& \left(\begin{array}{ll}
\alpha^{274} & \alpha^{237}
\end{array}\right)\left(\alpha^{169} \alpha^{342}\right)\left(\begin{array}{ll}
\alpha^{69} & \alpha^{442}
\end{array}\right)\left(\begin{array}{ll}
\alpha^{249} & \alpha^{262}
\end{array}\right)\left(\alpha^{71} \alpha^{440}\right)\left(\begin{array}{ll}
\alpha^{198} & \alpha^{313}
\end{array}\right)\left(\begin{array}{ll}
\alpha^{242} & \alpha^{269}
\end{array}\right) \\
& \left(\alpha^{73} \alpha^{438}\right)\left(\alpha^{146} \alpha^{365}\right)\left(\begin{array}{ll}
\alpha^{292} & \alpha^{219}
\end{array}\right)\left(\alpha^{74} \alpha^{437}\right)\left(\begin{array}{ll}
\alpha^{165} & \alpha^{346}
\end{array}\right)\left(\begin{array}{ll}
\alpha^{272} & \alpha^{239}
\end{array}\right)\left(\begin{array}{ll}
\alpha^{75} & \alpha^{436}
\end{array}\right)
\end{aligned}
$$

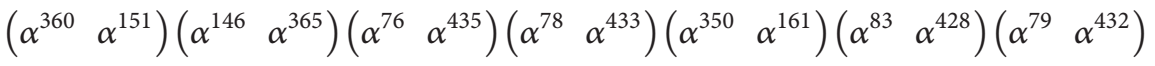

$$
\begin{aligned}
& \left(\begin{array}{ll}
\alpha^{228} & \alpha^{283}
\end{array}\right)\left(\begin{array}{ll}
\alpha^{204} & \alpha^{307}
\end{array}\right)\left(\begin{array}{ll}
\alpha^{81} & \alpha^{430}
\end{array}\right)\left(\begin{array}{ll}
\alpha^{298} & \alpha^{213}
\end{array}\right)\left(\begin{array}{ll}
\alpha^{132} & \alpha^{379}
\end{array}\right)\left(\begin{array}{ll}
\alpha^{84} & \alpha^{427}
\end{array}\right)\left(\begin{array}{ll}
\alpha^{118} & \alpha^{393}
\end{array}\right) \\
& \left(\alpha^{309} \alpha^{202}\right)\left(\alpha^{85} \alpha^{426}\right)\left(\alpha^{264} \alpha^{247}\right)\left(\alpha^{162} \alpha^{349}\right)\left(\alpha^{86} \alpha^{425}\right)\left(\alpha^{143} \alpha^{368}\right)\left(\alpha^{282} \alpha^{229}\right) \\
& \left(\begin{array}{ll}
\alpha^{87} & \alpha^{424}
\end{array}\right)\left(\begin{array}{ll}
\alpha^{267} & \alpha^{244}
\end{array}\right)\left(\begin{array}{ll}
\alpha^{157} & \alpha^{354}
\end{array}\right)\left(\begin{array}{ll}
\alpha^{88} & \alpha^{423}
\end{array}\right)\left(\alpha^{95} \alpha^{416}\right)\left(\begin{array}{ll}
\alpha^{328} & \alpha^{183}
\end{array}\right)\left(\begin{array}{ll}
\alpha^{89} & \alpha^{422}
\end{array}\right) \\
& \left(\begin{array}{ll}
\alpha^{325} & \alpha^{186}
\end{array}\right)\left(\alpha^{97} \alpha^{414}\right)\left(\alpha^{92} \alpha^{419}\right)\left(\begin{array}{ll}
\alpha^{234} & \alpha^{277}
\end{array}\right)\left(\begin{array}{ll}
\alpha^{185} & \alpha^{326}
\end{array}\right)\left(\begin{array}{ll}
\alpha^{93} & \alpha^{418}
\end{array}\right)\left(\begin{array}{ll}
\alpha^{211} & \alpha^{300}
\end{array}\right) \\
& \left(\begin{array}{ll}
\alpha^{207} & \alpha^{304}
\end{array}\right)\left(\begin{array}{ll}
\alpha^{98} & \alpha^{413}
\end{array}\right)\left(\begin{array}{ll}
\alpha^{140} & \alpha^{371}
\end{array}\right)\left(\alpha^{273} \alpha^{238}\right)\left(\alpha^{99} \alpha^{412}\right)\left(\begin{array}{ll}
\alpha^{121} & \alpha^{390}
\end{array}\right)\left(\begin{array}{ll}
\alpha^{291} & \alpha^{220}
\end{array}\right) \\
& \left(\alpha^{100} \alpha^{411}\right)\left(\begin{array}{ll}
\alpha^{124} & \alpha^{387}
\end{array}\right)\left(\begin{array}{ll}
\alpha^{287} & \alpha^{224}
\end{array}\right)\left(\alpha^{102} \alpha^{409}\right)\left(\begin{array}{ll}
\alpha^{295} & \alpha^{216}
\end{array}\right)\left(\begin{array}{ll}
\alpha^{114} & \alpha^{397}
\end{array}\right)\left(\begin{array}{ll}
\alpha^{105} & \alpha^{406}
\end{array}\right) \\
& \left(\alpha^{279} \alpha^{232}\right)\left(\alpha^{127} \alpha^{384}\right)\left(\alpha^{107} \alpha^{404}\right)\left(\alpha^{168} \alpha^{343}\right)\left(\alpha^{236} \alpha^{275}\right)\left(\alpha^{110} \alpha^{401}\right)\left(\alpha^{195} \alpha^{316}\right) \\
& \left(\alpha^{206} \alpha^{305}\right)\left(\alpha^{113} \alpha^{398}\right)\left(\begin{array}{ll}
\alpha^{245} & \alpha^{266}
\end{array}\right)\left(\alpha^{153} \alpha^{358}\right)\left(\alpha^{116} \alpha^{395}\right)\left(\alpha^{203} \alpha^{308}\right)\left(\alpha^{192} \alpha^{319}\right) \\
& \left(\begin{array}{ll}
\alpha^{122} & \alpha^{389}
\end{array}\right)\left(\begin{array}{ll}
\alpha^{212} & \alpha^{299}
\end{array}\right)\left(\begin{array}{ll}
\alpha^{177} & \alpha^{334}
\end{array}\right)\left(\begin{array}{ll}
\alpha^{123} & \alpha^{388}
\end{array}\right)\left(\alpha^{233} \alpha^{278}\right)\left(\begin{array}{ll}
\alpha^{155} & \alpha^{356}
\end{array}\right)\left(\begin{array}{ll}
\alpha^{128} & \alpha^{383}
\end{array}\right) \\
& \left(\alpha^{179} \alpha^{332}\right)\left(\alpha^{139} \alpha^{372}\right)\left(\alpha^{194} \alpha^{317}\right)\left(\alpha^{145} \alpha^{366}\right)\left(\alpha^{176} \alpha^{335}\right)\left(\begin{array}{ll}
\alpha^{190} & \alpha^{321}
\end{array}\right)\left(\begin{array}{ll}
\alpha^{150} & \alpha^{361}
\end{array}\right) \\
& c\left(\alpha^{209} \alpha^{302}\right)\left(\alpha^{152} \alpha^{359}\right)\left(\begin{array}{ll}
\alpha^{156} & \alpha^{355}
\end{array}\right)\left(\begin{array}{ll}
\alpha^{189} & \alpha^{322}
\end{array}\right)\left(\begin{array}{ll}
\alpha^{166} & \alpha^{345}
\end{array}\right)\left(\begin{array}{ll}
0 & \infty
\end{array}\right)(1) \\
& y:\left(\begin{array}{lll}
\alpha^{1} & \alpha^{129} & \alpha^{381}
\end{array}\right)\left(\begin{array}{lll}
\alpha^{510} & \alpha^{130} & \alpha^{382}
\end{array}\right)\left(\begin{array}{lll}
\alpha^{2} & \alpha^{258} & \alpha^{251}
\end{array}\right)\left(\begin{array}{llll}
\alpha^{509} & \alpha^{260} & \alpha^{253}
\end{array}\right)\left(\begin{array}{lll}
\alpha^{3} & \alpha^{417} & \alpha^{91}
\end{array}\right)\left(\begin{array}{lll}
\alpha^{508} & \alpha^{420} & \alpha^{94}
\end{array}\right) \\
& \left(\begin{array}{lll}
\alpha^{4} & \alpha^{5} & \alpha^{502}
\end{array}\right)\left(\begin{array}{lll}
\alpha^{507} & \alpha^{9} & \alpha^{506}
\end{array}\right)\left(\begin{array}{lll}
\alpha^{6} & \alpha^{323} & \alpha^{182}
\end{array}\right)\left(\begin{array}{lll}
\alpha^{505} & \alpha^{329} & \alpha^{188}
\end{array}\right)\left(\begin{array}{lll}
\alpha^{7} & \alpha^{60} & \alpha^{444}
\end{array}\right)\left(\begin{array}{lll}
\alpha^{504} & \alpha^{67} & \alpha^{451}
\end{array}\right) \\
& \left(\begin{array}{lll}
\alpha^{8} & \alpha^{10} & \alpha^{493}
\end{array}\right)\left(\begin{array}{lll}
\alpha^{503} & \alpha^{18} & \alpha^{501}
\end{array}\right)\left(\begin{array}{lll}
\alpha^{11} & \alpha^{459} & \alpha^{41}
\end{array}\right)\left(\begin{array}{lll}
\alpha^{500} & \alpha^{470} & \alpha^{52}
\end{array}\right)\left(\begin{array}{lll}
\alpha^{12} & \alpha^{135} & \alpha^{364}
\end{array}\right)\left(\begin{array}{lll}
\alpha^{499} & \alpha^{147} & \alpha^{376}
\end{array}\right) \\
& \left(\begin{array}{lll}
\alpha^{13} & \alpha^{125} & \alpha^{373}
\end{array}\right)\left(\begin{array}{lll}
\alpha^{498} & \alpha^{138} & \alpha^{386}
\end{array}\right)\left(\begin{array}{lll}
\alpha^{14} & \alpha^{120} & \alpha^{377}
\end{array}\right)\left(\begin{array}{lll}
\alpha^{497} & \alpha^{134} & \alpha^{391}
\end{array}\right)\left(\begin{array}{lll}
\alpha^{15} & \alpha^{111} & \alpha^{385}
\end{array}\right)\left(\begin{array}{lll}
\alpha^{496} & \alpha^{126} & \alpha^{400}
\end{array}\right) \\
& \left(\begin{array}{lll}
\alpha^{16} & \alpha^{20} & \alpha^{475}
\end{array}\right)\left(\begin{array}{lll}
\alpha^{495} & \alpha^{36} & \alpha^{491}
\end{array}\right)\left(\begin{array}{lll}
\alpha^{17} & \alpha^{324} & \alpha^{170}
\end{array}\right)\left(\begin{array}{lll}
\alpha^{494} & \alpha^{341} & \alpha^{187}
\end{array}\right)\left(\begin{array}{lll}
\alpha^{19} & \alpha^{402} & \alpha^{90}
\end{array}\right)\left(\begin{array}{lll}
\alpha^{492} & \alpha^{421} & \alpha^{109}
\end{array}\right) \\
& \left(\begin{array}{lll}
\alpha^{21} & \alpha^{285} & \alpha^{205}
\end{array}\right)\left(\begin{array}{lll}
\alpha^{490} & \alpha^{306} & \alpha^{226}
\end{array}\right)\left(\begin{array}{lll}
\alpha^{22} & \alpha^{407} & \alpha^{82}
\end{array}\right)\left(\begin{array}{lll}
\alpha^{489} & \alpha^{429} & \alpha^{104}
\end{array}\right)\left(\begin{array}{lll}
\alpha^{23} & \alpha^{314} & \alpha^{174}
\end{array}\right)\left(\begin{array}{lll}
\alpha^{488} & \alpha^{337} & \alpha^{197}
\end{array}\right) \\
& \left(\begin{array}{lll}
\alpha^{24} & \alpha^{270} & \alpha^{217}
\end{array}\right)\left(\begin{array}{lll}
\alpha^{487} & \alpha^{294} & \alpha^{241}
\end{array}\right)\left(\begin{array}{lll}
\alpha^{25} & \alpha^{31} & \alpha^{455}
\end{array}\right)\left(\begin{array}{lll}
\alpha^{486} & \alpha^{56} & \alpha^{480}
\end{array}\right)\left(\begin{array}{lll}
\alpha^{26} & \alpha^{250} & \alpha^{235}
\end{array}\right)\left(\begin{array}{lll}
\alpha^{485} & \alpha^{276} & \alpha^{261}
\end{array}\right) \\
& \left(\begin{array}{lll}
\alpha^{27} & \alpha^{115} & \alpha^{369}
\end{array}\right)\left(\begin{array}{lll}
\alpha^{484} & \alpha^{142} & \alpha^{396}
\end{array}\right)\left(\begin{array}{lll}
\alpha^{28} & \alpha^{240} & \alpha^{243}
\end{array}\right)\left(\begin{array}{llll}
\alpha^{483} & \alpha^{268} & \alpha^{271}
\end{array}\right)\left(\begin{array}{lll}
\alpha^{29} & \alpha^{434} & \alpha^{48}
\end{array}\right)\left(\begin{array}{lll}
\alpha^{482} & \alpha^{463} & \alpha^{77}
\end{array}\right) \\
& \left(\begin{array}{lll}
\alpha^{30} & \alpha^{222} & \alpha^{259}
\end{array}\right)\left(\begin{array}{lll}
\alpha^{481} & \alpha^{252} & \alpha^{289}
\end{array}\right)\left(\begin{array}{lll}
\alpha^{32} & \alpha^{40} & \alpha^{439}
\end{array}\right)\left(\begin{array}{lll}
\alpha^{479} & \alpha^{72} & \alpha^{471}
\end{array}\right)\left(\begin{array}{lll}
\alpha^{33} & \alpha^{148} & \alpha^{330}
\end{array}\right)\left(\begin{array}{lll}
\alpha^{478} & \alpha^{181} & \alpha^{363}
\end{array}\right) \\
& \left(\begin{array}{lll}
\alpha^{34} & \alpha^{137} & \alpha^{340}
\end{array}\right)\left(\begin{array}{lll}
\alpha^{477} & \alpha^{171} & \alpha^{374}
\end{array}\right)\left(\begin{array}{lll}
\alpha^{35} & \alpha^{196} & \alpha^{280}
\end{array}\right)\left(\begin{array}{lll}
\alpha^{476} & \alpha^{231} & \alpha^{315}
\end{array}\right)\left(\begin{array}{lll}
\alpha^{37} & \alpha^{338} & \alpha^{136}
\end{array}\right)\left(\begin{array}{lll}
\alpha^{474} & \alpha^{375} & \alpha^{173}
\end{array}\right) \\
& \left(\begin{array}{lll}
\alpha^{38} & \alpha^{293} & \alpha^{180}
\end{array}\right)\left(\begin{array}{lll}
\alpha^{473} & \alpha^{331} & \alpha^{218}
\end{array}\right)\left(\begin{array}{lll}
\alpha^{39} & \alpha^{175} & \alpha^{297}
\end{array}\right)\left(\begin{array}{lll}
\alpha^{472} & \alpha^{214} & \alpha^{336}
\end{array}\right)\left(\begin{array}{lll}
\alpha^{42} & \alpha^{59} & \alpha^{410}
\end{array}\right)\left(\begin{array}{ll}
\alpha^{469} & \alpha^{101} \\
\alpha^{452}
\end{array}\right) \\
& \left(\begin{array}{lll}
\alpha^{43} & \alpha^{327} & \alpha^{141}
\end{array}\right)\left(\begin{array}{lll}
\alpha^{468} & \alpha^{370} & \alpha^{184}
\end{array}\right)\left(\begin{array}{lll}
\alpha^{44} & \alpha^{303} & \alpha^{164}
\end{array}\right)\left(\begin{array}{lll}
\alpha^{467} & \alpha^{347} & \alpha^{208}
\end{array}\right)\left(\begin{array}{lll}
\alpha^{45} & \alpha^{265} & \alpha^{201}
\end{array}\right)\left(\begin{array}{lll}
\alpha^{466} & \alpha^{310} & \alpha^{246}
\end{array}\right) \\
& \left(\begin{array}{lll}
\alpha^{46} & \alpha^{117} & \alpha^{348}
\end{array}\right)\left(\begin{array}{lll}
\alpha^{465} & \alpha^{163} & \alpha^{394}
\end{array}\right)\left(\begin{array}{lll}
\alpha^{47} & \alpha^{254} & \alpha^{210}
\end{array}\right)\left(\begin{array}{lll}
\alpha^{464} & \alpha^{301} & \alpha^{257}
\end{array}\right)\left(\begin{array}{lll}
\alpha^{49} & \alpha^{70} & \alpha^{392}
\end{array}\right)\left(\begin{array}{ll}
\alpha^{462} & \alpha^{119} \\
\alpha^{441}
\end{array}\right) \\
& \left(\begin{array}{lll}
\alpha^{50} & \alpha^{62} & \alpha^{399}
\end{array}\right)\left(\begin{array}{lll}
\alpha^{461} & \alpha^{112} & \alpha^{449}
\end{array}\right)\left(\begin{array}{lll}
\alpha^{51} & \alpha^{403} & \alpha^{57}
\end{array}\right)\left(\begin{array}{lll}
\alpha^{460} & \alpha^{454} & \alpha^{108}
\end{array}\right)\left(\begin{array}{lll}
\alpha^{53} & \alpha^{172} & \alpha^{286}
\end{array}\right)\left(\begin{array}{lll}
\alpha^{458} & \alpha^{225} & \alpha^{339}
\end{array}\right)
\end{aligned}
$$




$$
\begin{aligned}
& \left(\begin{array}{lll}
\alpha^{54} & \alpha^{230} & \alpha^{227}
\end{array}\right)\left(\begin{array}{llll}
\alpha^{457} & \alpha^{284} & \alpha^{281}
\end{array}\right)\left(\begin{array}{llll}
\alpha^{55} & \alpha^{353} & \alpha^{103}
\end{array}\right)\left(\begin{array}{llll}
\alpha^{456} & \alpha^{408} & \alpha^{158}
\end{array}\right)\left(\begin{array}{lll}
\alpha^{58} & \alpha^{357} & \alpha^{96}
\end{array}\right)\left(\begin{array}{lll}
\alpha^{453} & \alpha^{415} & \alpha^{154}
\end{array}\right) \\
& \left(\begin{array}{lll}
\alpha^{61} & \alpha^{106} & \alpha^{344}
\end{array}\right)\left(\begin{array}{llll}
\alpha^{450} & \alpha^{167} & \alpha^{405}
\end{array}\right)\left(\begin{array}{llll}
\alpha^{63} & \alpha^{200} & \alpha^{248}
\end{array}\right)\left(\begin{array}{llll}
\alpha^{448} & \alpha^{263} & \alpha^{311}
\end{array}\right)\left(\begin{array}{llll}
\alpha^{64} & \alpha^{80} & \alpha^{367}
\end{array}\right)\left(\begin{array}{lll}
\alpha^{447} & \alpha^{144} & \alpha^{431}
\end{array}\right) \\
& \left(\begin{array}{lll}
\alpha^{65} & \alpha^{191} & \alpha^{255}
\end{array}\right)\left(\begin{array}{lll}
\alpha^{446} & \alpha^{256} & \alpha^{320}
\end{array}\right)\left(\begin{array}{lll}
\alpha^{66} & \alpha^{296} & \alpha^{149}
\end{array}\right)\left(\begin{array}{lll}
\alpha^{445} & \alpha^{362} & \alpha^{215}
\end{array}\right)\left(\begin{array}{lll}
\alpha^{68} & \alpha^{274} & \alpha^{169}
\end{array}\right)\left(\begin{array}{lll}
\alpha^{443} & \alpha^{342} & \alpha^{237}
\end{array}\right) \\
& \left(\begin{array}{lll}
\alpha^{69} & \alpha^{193} & \alpha^{249}
\end{array}\right)\left(\begin{array}{lll}
\alpha^{442} & \alpha^{262} & \alpha^{318}
\end{array}\right)\left(\begin{array}{lll}
\alpha^{71} & \alpha^{198} & \alpha^{242}
\end{array}\right)\left(\begin{array}{lll}
\alpha^{440} & \alpha^{269} & \alpha^{313}
\end{array}\right)\left(\begin{array}{lll}
\alpha^{73} & \alpha^{146} & \alpha^{292}
\end{array}\right)\left(\begin{array}{lll}
\alpha^{438} & \alpha^{219} & \alpha^{365}
\end{array}\right) \\
& \left(\begin{array}{lll}
\alpha^{74} & \alpha^{165} & \alpha^{272}
\end{array}\right)\left(\begin{array}{lll}
\alpha^{437} & \alpha^{239} & \alpha^{346}
\end{array}\right)\left(\begin{array}{llll}
\alpha^{75} & \alpha^{360} & \alpha^{76}
\end{array}\right)\left(\begin{array}{lll}
\alpha^{436} & \alpha^{435} & \alpha^{151}
\end{array}\right)\left(\begin{array}{lll}
\alpha^{78} & \alpha^{350} & \alpha^{83}
\end{array}\right)\left(\begin{array}{lll}
\alpha^{433} & \alpha^{428} & \alpha^{161}
\end{array}\right) \\
& \left(\begin{array}{lll}
\alpha^{79} & \alpha^{228} & \alpha^{204}
\end{array}\right)\left(\begin{array}{lll}
\alpha^{432} & \alpha^{307} & \alpha^{283}
\end{array}\right)\left(\begin{array}{lll}
\alpha^{81} & \alpha^{298} & \alpha^{132}
\end{array}\right)\left(\begin{array}{lll}
\alpha^{430} & \alpha^{379} & \alpha^{213}
\end{array}\right)\left(\begin{array}{lll}
\alpha^{84} & \alpha^{118} & \alpha^{309}
\end{array}\right)\left(\begin{array}{lll}
\alpha^{427} & \alpha^{202} & \alpha^{393}
\end{array}\right) \\
& \left(\begin{array}{lll}
\alpha^{85} & \alpha^{264} & \alpha^{162}
\end{array}\right)\left(\begin{array}{lll}
\alpha^{426} & \alpha^{349} & \alpha^{247}
\end{array}\right)\left(\begin{array}{llll}
\alpha^{86} & \alpha^{143} & \alpha^{282}
\end{array}\right)\left(\begin{array}{lll}
\alpha^{425} & \alpha^{229} & \alpha^{368}
\end{array}\right)\left(\begin{array}{lll}
\alpha^{87} & \alpha^{267} & \alpha^{157}
\end{array}\right)\left(\begin{array}{lll}
\alpha^{424} & \alpha^{354} & \alpha^{244}
\end{array}\right) \\
& \left(\begin{array}{lll}
\alpha^{88} & \alpha^{95} & \alpha^{328}
\end{array}\right)\left(\begin{array}{lll}
\alpha^{423} & \alpha^{183} & \alpha^{416}
\end{array}\right)\left(\begin{array}{llll}
\alpha^{89} & \alpha^{325} & \alpha^{97}
\end{array}\right)\left(\begin{array}{llll}
\alpha^{422} & \alpha^{414} & \alpha^{186}
\end{array}\right)\left(\begin{array}{llll}
\alpha^{92} & \alpha^{234} & \alpha^{185}
\end{array}\right)\left(\begin{array}{lll}
\alpha^{419} & \alpha^{326} & \alpha^{277}
\end{array}\right) \\
& \left(\begin{array}{lll}
\alpha^{93} & \alpha^{211} & \alpha^{207}
\end{array}\right)\left(\begin{array}{lll}
\alpha^{418} & \alpha^{304} & \alpha^{300}
\end{array}\right)\left(\begin{array}{lll}
\alpha^{98} & \alpha^{140} & \alpha^{273}
\end{array}\right)\left(\begin{array}{lll}
\alpha^{413} & \alpha^{238} & \alpha^{371}
\end{array}\right)\left(\begin{array}{lll}
\alpha^{99} & \alpha^{121} & \alpha^{291}
\end{array}\right)\left(\begin{array}{lll}
\alpha^{412} & \alpha^{220} & \alpha^{390}
\end{array}\right) \\
& \left(\begin{array}{lll}
\alpha^{100} & \alpha^{124} & \alpha^{287}
\end{array}\right)\left(\begin{array}{llll}
\alpha^{411} & \alpha^{224} & \alpha^{387}
\end{array}\right)\left(\begin{array}{llll}
\alpha^{102} & \alpha^{295} & \alpha^{114}
\end{array}\right)\left(\begin{array}{lll}
\alpha^{409} & \alpha^{397} & \alpha^{216}
\end{array}\right)\left(\begin{array}{lll}
\alpha^{105} & \alpha^{279} & \alpha^{127}
\end{array}\right)\left(\begin{array}{lll}
\alpha^{406} & \alpha^{384} & \alpha^{232}
\end{array}\right) \\
& \left(\begin{array}{lll}
\alpha^{107} & \alpha^{168} & \alpha^{236}
\end{array}\right)\left(\begin{array}{lll}
\alpha^{404} & \alpha^{275} & \alpha^{343}
\end{array}\right)\left(\begin{array}{llll}
\alpha^{110} & \alpha^{195} & \alpha^{206}
\end{array}\right)\left(\begin{array}{lll}
\alpha^{401} & \alpha^{305} & \alpha^{316}
\end{array}\right)\left(\begin{array}{lll}
\alpha^{113} & \alpha^{245} & \alpha^{153}
\end{array}\right)\left(\begin{array}{lll}
\alpha^{398} & \alpha^{358} & \alpha^{266}
\end{array}\right) \\
& \left(\begin{array}{lll}
\alpha^{116} & \alpha^{203} & \alpha^{192}
\end{array}\right)\left(\begin{array}{lll}
\alpha^{395} & \alpha^{319} & \alpha^{308}
\end{array}\right)\left(\begin{array}{lll}
\alpha^{122} & \alpha^{212} & \alpha^{177}
\end{array}\right)\left(\begin{array}{lll}
\alpha^{389} & \alpha^{334} & \alpha^{299}
\end{array}\right)\left(\begin{array}{lll}
\alpha^{123} & \alpha^{233} & \alpha^{155}
\end{array}\right)\left(\begin{array}{lll}
\alpha^{388} & \alpha^{356} & \alpha^{278}
\end{array}\right) \\
& \left(\begin{array}{lll}
\alpha^{128} & \alpha^{160} & \alpha^{223}
\end{array}\right)\left(\begin{array}{lll}
\alpha^{383} & \alpha^{288} & \alpha^{351}
\end{array}\right)\left(\begin{array}{llll}
\alpha^{131} & \alpha^{159} & \alpha^{221}
\end{array}\right)\left(\begin{array}{lll}
\alpha^{380} & \alpha^{290} & \alpha^{352}
\end{array}\right)\left(\begin{array}{lll}
\alpha^{133} & \alpha^{199} & \alpha^{179}
\end{array}\right)\left(\begin{array}{lll}
\alpha^{378} & \alpha^{332} & \alpha^{312}
\end{array}\right) \\
& c\left(\begin{array}{lll}
\alpha^{139} & \alpha^{194} & \alpha^{178}
\end{array}\right)\left(\begin{array}{lll}
\alpha^{372} & \alpha^{333} & \alpha^{317}
\end{array}\right)\left(\begin{array}{lll}
\alpha^{145} & \alpha^{176} & \alpha^{190}
\end{array}\right)\left(\begin{array}{lll}
\alpha^{366} & \alpha^{321} & \alpha^{335}
\end{array}\right)\left(\begin{array}{lll}
\alpha^{150} & \alpha^{209} & \alpha^{152}
\end{array}\right)\left(\begin{array}{lll}
\alpha^{361} & \alpha^{359} & \alpha^{302}
\end{array}\right) \\
& \left(\alpha^{156} \alpha^{189} \alpha^{166}\right)\left(\alpha^{355} \alpha^{345} \alpha^{322}\right)\left(\begin{array}{lll}
\infty & 1 & 0
\end{array}\right)
\end{aligned}
$$

The coset diagram for this action has 86 orbits (fragments). It has one copy of $\lambda$ (Figure 2) and 85 copies of $\gamma_{j}$ (Figure 3). First, we find the orbit of coset diagram which contains $\alpha^{1}$ (Figure 4).

In each $\gamma_{j}$, one can see that $x y x y^{-1} x \in \operatorname{PSL}(2, \mathbb{Z})$ is a path from the vertex $\alpha^{k}$ to the vertex $\alpha^{l}$, such that, during this journey, one has to pass each vertex of $\gamma_{j}$.

\section{Algebraic Structure of Proposed Substitution Box}

In the literature, algebraic techniques are being applied on Galois fields. In this novel technique, the initial sequence of Galois field is destroyed with the help of vertices of the coset diagram.

Let $G F^{*}\left(2^{9}\right)$ denote the elements of $G F\left(2^{9}\right)$ which are of the form of even powers of $\alpha$.

Our first aim is to write the vertices of coset diagram in a $16 \times 16$ matrix. For this, we choose only those vertices which belong to $G F^{*}\left(2^{9}\right)$.

Step 1. Our coset diagram has 86 orbits; we construct a $16 \times 16$ matrix having entries from $G F^{*}\left(2^{9}\right)$ in the following way.
First, we find the orbit of coset diagram which contains $\alpha^{1}$. Let us denote this orbit by $\gamma_{1}$ and apply $x y x y^{-1} x$ on $\alpha^{1}$, so that we reach $\alpha^{382}$. During this journey, we pass through $\alpha^{510}$, $\alpha^{130}, \alpha^{381}$, and $\alpha^{129}$ and at the end reach $\alpha^{382}$. Write $\alpha^{510}, \alpha^{130}$, and $\alpha^{382}$ as first three elements of the first row of $16 \times 16$ matrix.

After writing 3 vertices of any $\gamma_{k} \in\left\{\gamma_{j}: j=\right.$ $1,2,3, \ldots, 85\}$, in order to select the next copy from $\gamma_{j}$, we find a vertex $v=\alpha^{i_{1}+1}$, where $\alpha^{i_{1}}, \alpha^{i_{2}}, \alpha^{i_{3}}, \alpha^{i_{4}}, \alpha^{i_{5}}$, and $\alpha^{i_{6}}$ are the vertices of $\gamma_{k}$, such that $i_{1}<i_{j}$, where $j=2,3,4,5,6$. If $\alpha^{i_{1}+1}$ is already exhausted in previously chosen copies of $\gamma_{j}$, then we move to the copy of $\gamma_{j}$ containing $v=\alpha^{i_{1}+2}$ and so on. Apply $x y x y^{-1} x$ on $v$ so that we pass through each vertex. Note that, in each $\gamma_{j}$, only three vertices belong to $G F^{*}\left(2^{9}\right)$ out of 6 . Write these 3 vertices in $16 \times 16$ matrix in order. The process continues until all the 510 vertices of $\gamma_{j}$ are exhausted. Next, pick 0 from $\lambda$ and write it as last element of the last row.

Step 2. Consider $h: G F^{*}\left(2^{9}\right) \longrightarrow G F\left(2^{8}\right)$ defined by $f\left(\alpha^{2 n}\right)=$ $\omega^{n}$; the elements of $F_{2^{8}}$ can be represented by powers of $\omega$ (see Table 3), where $\omega$ is the root of irreducible polynomial $p(x)=x^{8}+x^{4}+x^{3}+x^{2}+1$ over $\mathbb{Z}_{2}$. In this step, we apply $h$ on each element of the matrix evolved in the first step. In this 


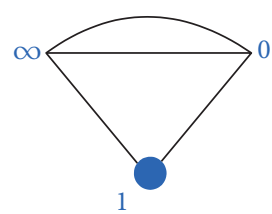

Figure 2: The orbit $\lambda$ of the coset diagram for the action of modular group on $G F\left(2^{9}\right) \cup\{\infty\}$.

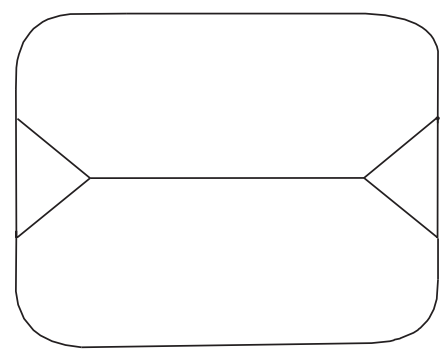

FIgURE 3: The orbit $\gamma_{j}$ of the coset diagram for the action of modular group on $G F\left(2^{9}\right) \cup\{\infty\}$.

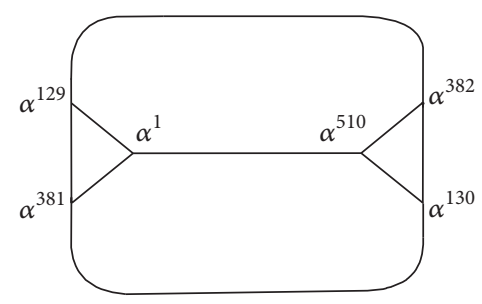

FIGURE 4: The copy of $\gamma_{j}$ having the vertex $\alpha^{1}$.

way, we obtain a $16 \times 16$ matrix having entries from $G F\left(2^{8}\right)$. Next, we convert each entry of the matrix into binary form and ultimately into decimal form (Table 4).

Before going to Step 3, let us define a map $g$ in the following way.

Let $f$ be the linear fractional transformation, obtained by the action of $P G L\left(2,\left(F\left(2^{8}\right)\right.\right.$ on $G F\left(2^{8}\right)$; that is, $f$ : $P G L\left(2, G F\left(2^{8}\right) \times G F\left(2^{8}\right)\right) \rightarrow G F\left(2^{8}\right)$.

Clearly $f$ is of the form $a z+b / c z+d$, where $a, b, c, d \in$ $G F\left(2^{8}\right)$, and is a mapping from $G F\left(2^{8}\right) \rightarrow G F\left(2^{8}\right)$. But, usually $f$ is not a bijective map.

(i) Let there be $n$ elements of $G F\left(2^{8}\right)$ missing in the range of $f$.

(ii) Let $s_{1}, s_{2}, s_{3}, \ldots, s_{k} \in G F\left(2^{8}\right)$ be repeated in the range of $f$.

(iii) Let $t_{1}, t_{2}, t_{3}, \ldots, t_{k}$ be the smallest elements in $G F\left(2^{8}\right)$ whose images are $s_{1}, s_{2}, s_{3}, \ldots, s_{k}$, respectively.

Suppose that $A=\left\{a_{1}, a_{2}, \ldots, a_{n}: a_{i}<a_{i+1}\right\}$ is the set of all those elements, except $t_{1}, t_{2}, t_{3}, \ldots, t_{k}$, whose images are $s_{1}, s_{2}, s_{3}, \ldots, s_{k}$, and $B=\left\{b_{1}, b_{2}, \ldots, b_{n}: b_{i}>b_{i+1}\right\}$ is the set of missing elements in the range of $f$. Then

$$
g(z)= \begin{cases}f(z) & \text { if } z \in G F\left(2^{8}\right)-A \\ b_{i} & \text { if } z=a_{i} .\end{cases}
$$

In this paper, we have taken $f(z)=214 z+93 / 124 z+123$.

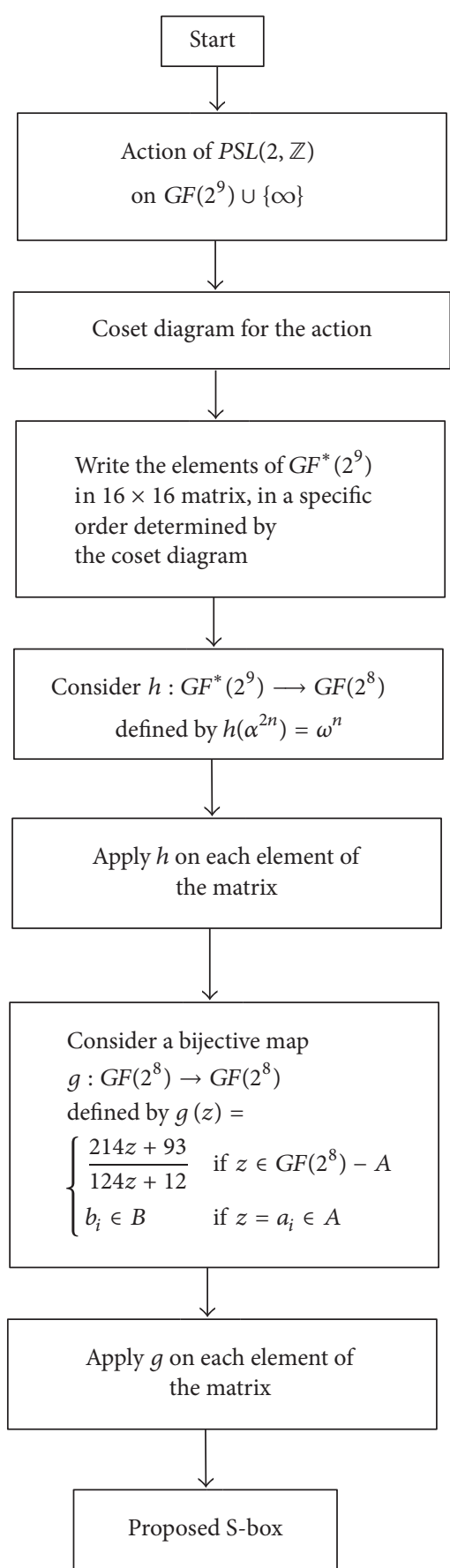

FIGURE 5: Flowchart for the construction of the proposed S-box.

Step 3. In this step, the bijective map $g$ defined by

$$
g(z)= \begin{cases}\frac{214 z+93}{124 z+123} & \text { if } z \in G F\left(2^{8}\right)-A \\ b_{i} & \text { if } z=a_{i}\end{cases}
$$

is applied on the matrix obtained at the end of Step 2 to evolve proposed S-box (Figure 5 and Table 5). More details of this last step are given in Table 2. 
TABLE 2: Construction of S-box using linear fractional transformation.

\begin{tabular}{|c|c|c|}
\hline$z$ & $f(z)=\frac{214 z+93}{124 z+123}$ & $g(z)$ \\
\hline 0 & $f(0)=\frac{93}{124}=\frac{01011101}{01111011}=\frac{\omega^{56}}{\omega^{172}}=\omega^{139}=1000010=66$ & 66 \\
\hline 1 & $f(1)=\frac{51}{247}=\frac{01110011}{11110111}=\frac{\omega}{\omega^{232}}=\omega^{148}=01010010=82$ & 82 \\
\hline 2 & $f(2)=\frac{9}{115}=\frac{00001001}{01110011}=\frac{\omega^{223}}{\omega^{159}}=\omega^{64}=01011111=95$ & 95 \\
\hline 254 & $f(254)=\frac{177}{131}=\frac{10110001}{10000011}=\frac{\omega^{86}}{\omega^{247}}=\omega^{94}=01110001=113$ & 2 \\
\hline 255 & $f(255)=\frac{135}{255}=\frac{10000111}{11111111}=\frac{\omega^{13}}{\omega^{175}}=\omega^{93}=10110110=182$ & 0 \\
\hline
\end{tabular}

TABLE 3: Representation of the elements of $G F\left(2^{8}\right)$.

\begin{tabular}{|c|c|c|c|c|c|c|c|}
\hline Binary values & $G F\left(2^{8}\right)$ & Binary values & $G F\left(2^{8}\right)$ & Binary values & $G F\left(2^{8}\right)$ & Binary values & $G F\left(2^{8}\right)$ \\
\hline 00000000 & 0 & 00000001 & 1 & 00000010 & $\omega^{1}$ & 00000100 & $\omega^{2}$ \\
\hline 00001000 & $\omega^{3}$ & 00010000 & $\omega^{4}$ & 00100000 & $\omega^{5}$ & 01000000 & $\omega^{6}$ \\
\hline 10000000 & $\omega^{7}$ & 00011101 & $\omega^{8}$ & 00111010 & $\omega^{9}$ & 01110100 & $\omega^{10}$ \\
\hline 11101000 & $\omega^{11}$ & 11001101 & $\omega^{12}$ & 10000111 & $\omega^{13}$ & 00010011 & $\omega^{14}$ \\
\hline 00100110 & $\omega^{15}$ & 01001100 & $\omega^{16}$ & 10011000 & $\omega^{17}$ & 00101101 & $\omega^{18}$ \\
\hline 01011010 & $\omega^{19}$ & 10110100 & $\omega^{20}$ & 01110101 & $\omega^{21}$ & 11101010 & $\omega^{22}$ \\
\hline 11001001 & $\omega^{23}$ & 10001111 & $\omega^{24}$ & 00000011 & $\omega^{25}$ & 00000110 & $\omega^{26}$ \\
\hline 00001100 & $\omega^{27}$ & 00011000 & $\omega^{28}$ & 00110000 & $\omega^{29}$ & 01100000 & $\omega^{30}$ \\
\hline 11000000 & $\omega^{31}$ & 10011101 & $\omega^{32}$ & 00100111 & $\omega^{33}$ & 01001110 & $\omega^{34}$ \\
\hline 10011100 & $\omega^{35}$ & 00100101 & $\omega^{36}$ & 01001010 & $\omega^{37}$ & 10010100 & $\omega^{38}$ \\
\hline 00110101 & $\omega^{39}$ & 01101010 & $\omega^{40}$ & 11010100 & $\omega^{41}$ & 10110101 & $\omega^{42}$ \\
\hline 01110111 & $\omega^{43}$ & 11101110 & $\omega^{44}$ & 11000001 & $\omega^{45}$ & 10011111 & $\omega^{46}$ \\
\hline 00100011 & $\omega^{47}$ & 01000110 & $\omega^{48}$ & 10001100 & $\omega^{49}$ & 00000101 & $\omega^{50}$ \\
\hline 00001010 & $\omega^{51}$ & 00010100 & $\omega^{52}$ & 00101000 & $\omega^{53}$ & 01010000 & $\omega^{54}$ \\
\hline 10100000 & $\omega^{55}$ & 01011101 & $\omega^{56}$ & 10111010 & $\omega^{57}$ & 01101001 & $\omega^{58}$ \\
\hline 11010010 & $\omega^{59}$ & 10111001 & $\omega^{60}$ & 01101111 & $\omega^{61}$ & 11011110 & $\omega^{62}$ \\
\hline 10100001 & $\omega^{63}$ & 01011111 & $\omega^{64}$ & 10111110 & $\omega^{65}$ & 01100001 & $\omega^{66}$ \\
\hline 11000010 & $\omega^{67}$ & 10011001 & $\omega^{68}$ & 00101111 & $\omega^{69}$ & 01011110 & $\omega^{70}$ \\
\hline 10111100 & $\omega^{71}$ & 01100101 & $\omega^{72}$ & 11001010 & $\omega^{73}$ & 10001001 & $\omega^{74}$ \\
\hline 00001111 & $\omega^{75}$ & 00011110 & $\omega^{76}$ & 00111100 & $\omega^{77}$ & 01111000 & $\omega^{78}$ \\
\hline 11110000 & $\omega^{79}$ & 11111101 & $\omega^{80}$ & 11100111 & $\omega^{81}$ & 11010011 & $\omega^{82}$ \\
\hline 10111011 & $\omega^{83}$ & 01101011 & $\omega^{84}$ & 11010110 & $\omega^{85}$ & 10110001 & $\omega^{86}$ \\
\hline 01111111 & $\omega^{87}$ & 11111110 & $\omega^{88}$ & 11100001 & $\omega^{89}$ & 11011111 & $\omega^{90}$ \\
\hline 10100011 & $\omega^{91}$ & 01011011 & $\omega^{92}$ & 10110110 & $\omega^{93}$ & 01110001 & $\omega^{94}$ \\
\hline 11100010 & $\omega^{95}$ & 11011001 & $\omega^{96}$ & 10101111 & $\omega^{97}$ & 01000011 & $\omega^{98}$ \\
\hline 10000110 & $\omega^{99}$ & 00010001 & $\omega^{100}$ & 00100010 & $\omega^{101}$ & 01000100 & $\omega^{102}$ \\
\hline 10001000 & $\omega^{103}$ & 00001101 & $\omega^{104}$ & 00011010 & $\omega^{105}$ & 00110100 & $\omega^{106}$ \\
\hline 01101000 & $\omega^{107}$ & 11010000 & $\omega^{108}$ & 10111101 & $\omega^{109}$ & 01100111 & $\omega^{110}$ \\
\hline 11001110 & $\omega^{111}$ & 10000001 & $\omega^{112}$ & 00011111 & $\omega^{113}$ & 00111110 & $\omega^{114}$ \\
\hline 01111100 & $\omega^{115}$ & 11111000 & $\omega^{116}$ & 11101101 & $\omega^{117}$ & 11000111 & $\omega^{118}$ \\
\hline 10010011 & $\omega^{119}$ & 00111011 & $\omega^{120}$ & 01110110 & $\omega^{121}$ & 11101100 & $\omega^{122}$ \\
\hline 11000101 & $\omega^{123}$ & 10010111 & $\omega^{124}$ & 00110011 & $\omega^{125}$ & 01100110 & $\omega^{126}$ \\
\hline 11001100 & $\omega^{127}$ & 10000101 & $\omega^{128}$ & 00010111 & $\omega^{129}$ & 00101110 & $\omega^{130}$ \\
\hline 01011100 & $\omega^{131}$ & 10111000 & $\omega^{132}$ & 01101101 & $\omega^{133}$ & 11011010 & $\omega^{134}$ \\
\hline
\end{tabular}


TABle 3: Continued.

\begin{tabular}{|c|c|c|c|c|c|c|c|}
\hline Binary values & $G F\left(2^{8}\right)$ & Binary values & $G F\left(2^{8}\right)$ & Binary values & $G F\left(2^{8}\right)$ & Binary values & $G F\left(2^{8}\right)$ \\
\hline 10101001 & $\omega^{135}$ & 01001111 & $\omega^{136}$ & 10011110 & $\omega^{137}$ & 00100001 & $\omega^{138}$ \\
\hline 01000010 & $\omega^{139}$ & 10000100 & $\omega^{140}$ & 00010101 & $\omega^{141}$ & 00101010 & $\omega^{142}$ \\
\hline 01010100 & $\omega^{143}$ & 10101000 & $\omega^{144}$ & 01001101 & $\omega^{145}$ & 10011010 & $\omega^{146}$ \\
\hline 00101001 & $\omega^{147}$ & 01010010 & $\omega^{148}$ & 10100100 & $\omega^{149}$ & 01010101 & $\omega^{150}$ \\
\hline 10101010 & $\omega^{151}$ & 01001001 & $\omega^{152}$ & 10010010 & $\omega^{153}$ & 00111001 & $\omega^{154}$ \\
\hline 01110010 & $\omega^{155}$ & 11100100 & $\omega^{156}$ & 11010101 & $\omega^{157}$ & 10110111 & $\omega^{158}$ \\
\hline 01110011 & $\omega^{159}$ & 11100110 & $\omega^{160}$ & 11010001 & $\omega^{161}$ & 10111111 & $\omega^{162}$ \\
\hline 01100011 & $\omega^{163}$ & 11000110 & $\omega^{164}$ & 10010001 & $\omega^{165}$ & 00111111 & $\omega^{166}$ \\
\hline 01111110 & $\omega^{167}$ & 11111100 & $\omega^{168}$ & 11100101 & $\omega^{169}$ & 11010111 & $\omega^{170}$ \\
\hline 10110011 & $\omega^{171}$ & 01111011 & $\omega^{172}$ & 11110110 & $\omega^{173}$ & 11110001 & $\omega^{174}$ \\
\hline 11111111 & $\omega^{175}$ & 11100011 & $\omega^{176}$ & 11011011 & $\omega^{177}$ & 10101011 & $\omega^{178}$ \\
\hline 01001011 & $\omega^{179}$ & 10010110 & $\omega^{180}$ & 00110001 & $\omega^{181}$ & 01100010 & $\omega^{182}$ \\
\hline 11000100 & $\omega^{183}$ & 10010101 & $\omega^{184}$ & 00110111 & $\omega^{185}$ & 01101110 & $\omega^{186}$ \\
\hline 11011100 & $\omega^{187}$ & 10100101 & $\omega^{188}$ & 01010111 & $\omega^{189}$ & 10101110 & $\omega^{190}$ \\
\hline 01000001 & $\omega^{191}$ & 10000010 & $\omega^{192}$ & 00011001 & $\omega^{193}$ & 00110010 & $\omega^{194}$ \\
\hline 01100100 & $\omega^{195}$ & 11001000 & $\omega^{196}$ & 10001101 & $\omega^{197}$ & 00000111 & $\omega^{198}$ \\
\hline 00001110 & $\omega^{199}$ & 00011100 & $\omega^{200}$ & 00111000 & $\omega^{201}$ & 01110000 & $\omega^{202}$ \\
\hline 11100000 & $\omega^{203}$ & 11011101 & $\omega^{204}$ & 10100111 & $\omega^{205}$ & 01010011 & $\omega^{206}$ \\
\hline 10100110 & $\omega^{207}$ & 01010001 & $\omega^{208}$ & 10100010 & $\omega^{209}$ & 01011001 & $\omega^{210}$ \\
\hline 10110010 & $\omega^{211}$ & 01111001 & $\omega^{212}$ & 11110010 & $\omega^{213}$ & 11111001 & $\omega^{214}$ \\
\hline 11101111 & $\omega^{215}$ & 11000011 & $\omega^{216}$ & 10011011 & $\omega^{217}$ & 00101011 & $\omega^{218}$ \\
\hline 01010110 & $\omega^{219}$ & 10101100 & $\omega^{220}$ & 01000101 & $\omega^{221}$ & 10001010 & $\omega^{222}$ \\
\hline 00001001 & $\omega^{223}$ & 00010010 & $\omega^{224}$ & 00100100 & $\omega^{225}$ & 01001000 & $\omega^{226}$ \\
\hline 10010000 & $\omega^{227}$ & 00111101 & $\omega^{228}$ & 01111010 & $\omega^{229}$ & 11110100 & $\omega^{230}$ \\
\hline 11110101 & $\omega^{231}$ & 11110111 & $\omega^{232}$ & 11110011 & $\omega^{233}$ & 11111011 & $\omega^{234}$ \\
\hline 11101011 & $\omega^{235}$ & 11001011 & $\omega^{236}$ & 10001011 & $\omega^{237}$ & 00001011 & $\omega^{238}$ \\
\hline 00010110 & $\omega^{239}$ & 00101100 & $\omega^{240}$ & 01011000 & $\omega^{241}$ & 10110000 & $\omega^{242}$ \\
\hline 01111101 & $\omega^{243}$ & 11111010 & $\omega^{244}$ & 11101001 & $\omega^{245}$ & 11001111 & $\omega^{246}$ \\
\hline 10000011 & $\omega^{247}$ & 00011011 & $\omega^{248}$ & 00110110 & $\omega^{249}$ & 01101100 & $\omega^{250}$ \\
\hline 11011000 & $\omega^{251}$ & 10101101 & $\omega^{252}$ & 01000111 & $\omega^{253}$ & 10001110 & $\omega^{254}$ \\
\hline
\end{tabular}

TABLE 4: $16 \times 16$ matrix evolved after 2 nd step.

\begin{tabular}{|c|c|c|c|c|c|c|c|c|c|c|c|c|c|c|c|}
\hline 1 & 190 & 65 & 2 & 23 & 46 & 142 & 89 & 35 & 4 & 216 & 71 & 8 & 163 & 113 & 96 \\
\hline 138 & 173 & 16 & 32 & 58 & 108 & 235 & 6 & 64 & 98 & 165 & 54 & 47 & 25 & 128 & 185 \\
\hline 194 & 27 & 161 & 28 & 29 & 116 & 45 & 191 & 214 & 131 & 56 & 193 & 207 & 233 & 146 & 31 \\
\hline 232 & 212 & 20 & 213 & 127 & 250 & 205 & 169 & 41 & 125 & 24 & 44 & 135 & 51 & 33 & 176 \\
\hline 188 & 7 & 19 & 59 & 218 & 155 & 143 & 88 & 38 & 206 & 102 & 76 & 180 & 37 & 137 & 145 \\
\hline 22 & 152 & 215 & 220 & 67 & 132 & 11 & 229 & 153 & 139 & 90 & 223 & 189 & 203 & 104 & 252 \\
\hline 117 & 167 & 72 & 251 & 55 & 91 & 234 & 211 & 13 & 243 & 114 & 197 & 201 & 241 & 141 & 204 \\
\hline 26 & 247 & 156 & 200 & 245 & 3 & 192 & 93 & 244 & 144 & 80 & 177 & 84 & 122 & 12 & 124 \\
\hline 42 & 61 & 221 & 240 & 48 & 70 & 60 & 40 & 123 & 36 & 17 & 151 & 18 & 157 & 106 & 101 \\
\hline 9 & 133 & 230 & 39 & 82 & 49 & 78 & 158 & 179 & 69 & 92 & 115 & 134 & 118 & 172 & 202 \\
\hline 154 & 86 & 74 & 79 & 246 & 150 & 148 & 43 & 53 & 255 & 249 & 62 & 68 & 195 & 164 & 97 \\
\hline 239 & 181 & 210 & 34 & 184 & 231 & 242 & 119 & 21 & 149 & 121 & 219 & 236 & 238 & 198 & 81 \\
\hline 178 & 166 & 182 & 159 & 237 & 99 & 162 & 73 & 85 & 140 & 94 & 147 & 83 & 103 & 100 & 5 \\
\hline 222 & 129 & 10 & 186 & 208 & 224 & 130 & 248 & 107 & 199 & 112 & 160 & 136 & 183 & 14 & 75 \\
\hline 109 & 105 & 217 & 57 & 111 & 52 & 126 & 50 & 171 & 66 & 95 & 253 & 168 & 174 & 77 & 227 \\
\hline 87 & 63 & 228 & 175 & 225 & 110 & 254 & 226 & 196 & 15 & 30 & 170 & 120 & 187 & 209 & 0 \\
\hline
\end{tabular}


TABle 5: Proposed S-box evolved after 3rd step.

\begin{tabular}{lccccccccccccccc}
\hline 82 & 114 & 50 & 95 & 120 & 28 & 196 & 91 & 44 & 242 & 70 & 153 & 205 & 147 & 73 & 40 \\
\hline 204 & 136 & 250 & 48 & 247 & 166 & 29 & 183 & 157 & 237 & 145 & 243 & 53 & 122 & 225 & 121 \\
109 & 65 & 149 & 253 & 221 & 144 & 203 & 112 & 76 & 219 & 93 & 110 & 88 & 33 & 181 & 63 \\
34 & 79 & 171 & 78 & 182 & 7 & 92 & 140 & 213 & 227 & 132 & 160 & 210 & 52 & 174 & 133 \\
116 & 226 & 47 & 234 & 67 & 162 & 191 & 151 & 155 & 90 & 42 & 233 & 127 & 246 & 207 & 185 \\
71 & 175 & 72 & 61 & 13 & 217 & 39 & 37 & 169 & 202 & 80 & 57 & 115 & 97 & 9 & 5 \\
55 & 142 & 26 & 6 & 30 & 74 & 32 & 81 & 4 & 17 & 86 & 105 & 99 & 19 & 197 & 94 \\
75 & 12 & 161 & 100 & 15 & 46 & 111 & 235 & 16 & 188 & 208 & 130 & 69 & 163 & 255 & 229 \\
244 & 164 & 60 & 21 & 230 & 49 & 154 & 223 & 54 & 212 & 195 & 176 & 165 & 159 & 184 & 102 \\
64 & 214 & 36 & 252 & 59 & 117 & 194 & 158 & 128 & 240 & 11 & 45 & 211 & 198 & 137 & 98 \\
167 & 168 & 224 & 239 & 14 & 177 & 179 & 1 & 77 & 0 & 8 & 87 & 89 & 108 & 146 & 248 \\
22 & 126 & 83 & 193 & 123 & 35 & 18 & 186 & 96 & 178 & 199 & 62 & 25 & 23 & 104 & 206 \\
129 & 143 & 125 & 152 & 24 & 20 & 148 & 27 & 218 & 200 & 238 & 180 & 190 & 232 & 216 & 228 \\
58 & 222 & 189 & 119 & 85 & 56 & 220 & 10 & 103 & 101 & 173 & 150 & 209 & 124 & 254 & 107 \\
231 & 31 & 68 & 170 & 236 & 251 & 113 & 245 & 138 & 215 & 192 & 3 & 141 & 135 & 172 & 41 \\
187 & 201 & 38 & 134 & 51 & 131 & 2 & 43 & 106 & 241 & 249 & 139 & 156 & 118 & 84 & 66 \\
\hline
\end{tabular}

TABLE 6: Nonlinearity of basic functions of various substitution boxes.

\begin{tabular}{|c|c|c|c|c|c|c|c|c|c|}
\hline S-box & $f_{0}$ & $f_{1}$ & $f_{2}$ & $f_{3}$ & $f_{4}$ & $f_{5}$ & $f_{6}$ & $f_{7}$ & Ave \\
\hline Gray [13] & 112 & 112 & 112 & 112 & 112 & 112 & 112 & 112 & 112 \\
\hline Gautam et al. [14] & 108 & 106 & 104 & 98 & 102 & 102 & 98 & 74 & 99 \\
\hline Prime [15] & 94 & 100 & 104 & 104 & 102 & 100 & 98 & 94 & 99.5 \\
\hline $\mathrm{S}_{8}$ AES [16] & 112 & 112 & 112 & 112 & 112 & 112 & 112 & 112 & 112 \\
\hline Shi et al. [17] & 106 & 104 & 106 & 106 & 104 & 106 & 104 & 106 & 105 \\
\hline AES [2] & 112 & 112 & 112 & 112 & 112 & 112 & 112 & 112 & 112 \\
\hline Skipjac and Kea [18] & 104 & 108 & 108 & 108 & 108 & 104 & 104 & 106 & 106.75 \\
\hline Alkhaldi et al. [19] & 108 & 104 & 106 & 106 & 102 & 98 & 104 & 108 & 104 \\
\hline Chen et al. [20] & 100 & 102 & 103 & 104 & 106 & 106 & 106 & 108 & 104.3 \\
\hline Tang et al. [21] & 100 & 103 & 104 & 104 & 105 & 105 & 106 & 109 & 104.5 \\
\hline Khan et al. [22] & 102 & 108 & 106 & 102 & 106 & 106 & 106 & 98 & 104.25 \\
\hline Belazi et al. [23] & 106 & 106 & 106 & 104 & 108 & 102 & 106 & 104 & 105.25 \\
\hline Proposed & 108 & 106 & 108 & 108 & 108 & 104 & 106 & 106 & 106.75 \\
\hline
\end{tabular}

\section{Statistical Analysis and Simulation Results}

In this section, we implement various security performance tests on newly created S-box to examine its special properties. The assessment of the characteristics of proposed S-box determines its application in different encryption methods and for security purposes. We use five different security performance tests, namely, linear approximation probability (LP), differential approximation probability (DP), nonlinearity, bit independence criterion (BIC), and strict avalanche criterion, to assess the cryptographic competence of substitution box. The results obtained from proposed Sbox are then compared with the well-known S-boxes. The description of different types of tests implemented on these $\mathrm{S}$-boxes is given below.
5.1. Nonlinearity. The concept of nonlinearity was first introduced by Pieprzyk and Finkelstein in 1988 [8]. It is the basic tool to measure the strength of the S-box. An S-box with bigger nonlinearity is more secure than that with lesser nonlinearity. The nonlinearity is expressed as

$$
N_{h}=2^{n-1}\left(1-2^{-n} \max \left|S_{\langle h\rangle}(\alpha)\right|\right),
$$

where $S_{\langle h\rangle}(\alpha)=\sum(-1)^{h(\beta) \oplus \beta \cdot \alpha}$ is Walsh spectrum and $\alpha, \beta \epsilon$ $G F\left(2^{n}\right)$.

The average value of the nonlinearity of the proposed Sbox is 106.75. In Table 6, nonlinearity of the proposed S-box is compared with multiple renowned substitution boxes. One can see that the nonlinearity of the proposed S-box is better than most of the familiar S-boxes. 
TABLE 7: Nonlinearity of bit independence criterion of the proposed S-box.

\begin{tabular}{|c|c|c|c|c|c|c|c|}
\hline- & 96 & 104 & 104 & 106 & 106 & 108 & 106 \\
\hline 96 & - & 106 & 102 & 102 & 102 & 106 & 106 \\
\hline 104 & 106 & - & 104 & 102 & 106 & 102 & 106 \\
\hline 104 & 102 & 104 & - & 104 & 102 & 104 & 102 \\
\hline 106 & 102 & 102 & 104 & - & 104 & 104 & 96 \\
\hline 106 & 102 & 106 & 102 & 104 & - & 102 & 106 \\
\hline 108 & 106 & 102 & 104 & 104 & 102 & - & 104 \\
\hline 106 & 106 & 106 & 102 & 96 & 106 & 104 & - \\
\hline
\end{tabular}

TABLE 8: Bit independence criterion of various substitution boxes.

\begin{tabular}{lccc}
\hline S-boxes & Minimum value & Average & Square deviation \\
\hline Proposed & 96 & 103.643 & 2.7283 \\
Gray [13] & 112 & 112 & 0 \\
Gautam et al. [14] & 92 & 103 & 3.5225 \\
Prime [15] & 94 & 101.71 & 3.53 \\
S $_{8}$ AES [16] & 112 & 112 & 0 \\
Hussain et al. [12] & 98 & 103.78 & 2.743 \\
AES [2] & 112 & 112 & 0 \\
Hussain et al. [15] & 102 & 104.14 & 1.767 \\
\hline
\end{tabular}

TABLE 9: Strict avalanche criterion of the proposed S-box.

\begin{tabular}{llllllll}
\hline .4375 & .5000 & .5000 & .4844 & .4844 & .4844 & .5312 & .4375 \\
\hline .5000 & .4375 & .5000 & .5625 & .5000 & .5469 & .4688 & .5000 \\
.5312 & .5469 & .5156 & .4687 & .4531 & .5312 & .5156 & .4375 \\
.4844 & .4375 & .5469 & .5312 & .4688 & .5781 & .5781 & .5000 \\
.4688 & .5156 & .4531 & .5625 & .5781 & .5469 & .4844 & .5156 \\
.5000 & .5156 & .5312 & .5000 & .4688 & .5156 & .5488 & .5000 \\
.5156 & .5313 & .4844 & .4687 & .4531 & .5000 & .4688 & .4531 \\
.5312 & .4844 & .5625 & .5469 & .4844 & .4531 & .5625 \\
\hline
\end{tabular}

5.2. Bit Independence Criterion. According to bit independence criterion $[9,10]$, if any input bit $i$ is inverted, then the output bits $j$ and $k$ must change independently. In other words, the avalanche variables must be pairwise independent for a given set of avalanche vectors. We have tested the nonlinearity of bit independence criterion of S-box (Table 7). We also compared the minimum and average values of bit independence criterion along with square deviation of the proposed S-box with different renowned S-boxes (Table 8).

5.3. Strict Avalanche Criterion. Strict avalanche criterion (SAC) introduced by Tavares and Webster is founded on the ideas of the avalanche and completeness effect $[9,10]$. It is a formalization of the avalanche effect. If by complementing a single input bit all the output bits are changed with a 0.5 probability, SAC is said to be satisfied. Table 9 displays the outcomes of the strict avalanche criterion.
5.4. Linear Approximation Probability. In linear approximation probability method, we examine the imbalance of an event [11]. The analysis is used to calculate the highest value of imbalance of the outcome of the event. The uniformity of the input bits should be similar to that of the output bits. Each $i$ th input bit is analyzed individually and its outcomes are checked in the output bits. The masks which are applied on the parity of both input and output bits are denoted by $\chi_{x}$ and $\chi_{y}$, respectively. Mathematically,

$$
\mathrm{LP}=\max _{\chi_{x}, \chi_{y} \neq 0}\left|\frac{\#\left\{d / d \cdot \chi_{x}=S(d) \cdot \chi_{y}\right\}}{2^{n}}-\frac{1}{2}\right| \text {, }
$$

where $d$ represents the collection of all possible inputs and $2^{n}$ is the total number of elements. The results of this important 
TABLE 10: Linear approximation probability analyses of different S-boxes.

\begin{tabular}{lcccccccc}
\hline S-boxes & AES & Gray & Skipjack & Prime & Proposed & Gautam et al. [14] & S $_{8}$ AES & Xyi \\
\hline Max value & 144 & 144 & 156 & 162 & 162 & 164 & 144 \\
Max LP & 0.062 & 0.062 & 0.109 & 0.132 & 0.1484 & 0.2109 & 0.062 & 0.156 \\
\hline
\end{tabular}

TABLE 11: Differential probability of the proposed S-box.

\begin{tabular}{lllllllllllllllll}
\hline .02344 & .02344 & .02344 & .02344 & .03125 & .02344 & .02344 & .02344 & .02344 & .03125 & .02344 & .02344 & .02344 & .01562 & .02344 & .03125 \\
\hline .02344 & .02344 & .03125 & .03125 & .03125 & .03125 & .03125 & .02344 & .02344 & .03125 & .02344 & .02344 & .02344 & .02344 & .02344 & .02344 \\
.01563 & .02344 & .02344 & .03125 & .03125 & .03125 & .02344 & .02344 & .02344 & .02344 & .03125 & .02344 & .02344 & .02344 & .01562 & .02344 \\
.03125 & .03906 & .02344 & .03125 & .02344 & .02344 & .03125 & .02344 & .03125 & .03125 & .03125 & .02344 & .02344 & .02344 & .02344 & .02344 \\
.03125 & .04688 & .03906 & .02344 & .02344 & .02344 & .03125 & .02344 & .01562 & .02344 & .03125 & .02344 & .02344 & .02344 & .02344 & .03125 \\
.03125 & .03125 & .02344 & .03125 & .03125 & .03125 & .03125 & .03125 & .02344 & .02344 & .02344 & .03125 & .02344 & .03125 & .02344 & .02344 \\
.02344 & .03125 & .03125 & .03906 & .03125 & .02344 & .03125 & .03906 & .02344 & .02344 & .03125 & .02344 & .03125 & .02344 & .03125 & .02344 \\
.01563 & .03125 & .03125 & .03125 & .03125 & .03125 & .02344 & .02344 & .03125 & .03125 & .02344 & .03125 & .02344 & .03125 & .02344 & .02344 \\
.03125 & .03125 & .03906 & .02344 & .03125 & .02344 & .02344 & .03125 & .03125 & .03125 & .02344 & .03125 & .02344 & .03906 & .03125 & .03125 \\
.03125 & .02344 & .03125 & .02344 & .03125 & .03125 & .02344 & .03125 & .02344 & .02344 & .02344 & .03125 & .02344 & .03125 & .02344 & .02344 \\
.02344 & .03125 & .02344 & .02344 & .02344 & .03125 & .03125 & .02344 & .03125 & .02344 & .02344 & .03125 & .02344 & .02344 & .02344 & .03125 \\
.02344 & .02344 & .03125 & .02344 & .02344 & .02344 & .02344 & .02344 & .02344 & .02344 & .03125 & .03125 & .02344 & .02344 & .02344 & .02344 \\
.02344 & .02344 & .02344 & .02344 & .02344 & .02344 & .03125 & .02344 & .02344 & .02344 & .03125 & .02344 & .02344 & .03125 & .03125 & .03125 \\
.03125 & .02344 & .04688 & .02344 & .02344 & .02344 & .03906 & .03125 & .03125 & .02344 & .02344 & .02344 & .03125 & .03125 & .02344 & .03125 \\
.02344 & .02344 & .03906 & .03125 & .02344 & .02344 & .02344 & .02344 & .02344 & .03125 & .03125 & .02344 & .03125 & .02344 & .03906 & .03125 \\
.03125 & .03125 & .02344 & .02344 & .03125 & .03125 & .02344 & .02344 & .02344 & .03125 & .02344 & .02344 & .02344 & .03125 & .02344 & - \\
\hline
\end{tabular}

analysis obtained from our S-box and different established Sboxes are given in Table 10. The comparison shows that our S-box is strong enough to deal with different linear attacks.

5.5. Differential Approximation Probability. In this analysis, differential uniformity is determined by examining the mapping from the input bits to the output. The main focus of this test is to ensure differential uniformity; that is, the input differential must be associated with an output differential in a unique way.

It is represented by

$$
\begin{aligned}
D_{P^{s}} & (\Delta \mu \longrightarrow \Delta \nu) \\
& =\frac{[\#\{\mu \varepsilon I / S(\mu) \bigoplus S(\mu \oplus \Delta \mu)=\Delta \nu\}]}{2^{n}},
\end{aligned}
$$

where $\Delta \mu$ and $\Delta \nu$ are the input and output differentials, respectively. We have applied differential approximation probability test on our S-box. The results are presented in Table 11.

\section{Majority Logic Criterion}

The majority logic criterion [12] is helpful in finding the premier candidate S-box fit for a certain kind of encryption application. In this criterion, image encryption strength of the
S-box is investigated through statistical studies. A distortion in the image is created by encryption process; therefore, it is important to study the statistical characteristics. It is achieved with the help of various analyses such as entropy, contrast, correlation, energy, and homogeneity. The proposed S-box can further be used for encryption and multimedia security. In this paper, we have used two JPEG images, Pepper and Baboon, for MLC analysis. The results of these analyses in comparison with the other well-known S-boxes are depicted in Table 12. Figure 6 shows the result of image encryption with proposed S-box. The histogram of the original image and the encrypted images of Baboon and Pepper are shown in Figure 7. These results show that our S-box fulfills all the requirements to be declared as a very suitable S-box for encryption applications. Thus, it is recommended to become a part of algorithms designed for the secure transmission of information/data.

\section{Conclusion}

In the present study, a strong S-box is created with the help of coset graph for the action of modular group and a bijective map. According to our information, this is the first use of coset graphs in the construction of S-box. The proposed Sbox is highly secure and the results obtained from different analyses are nearly equal to the ideal ones. Therefore, it is very useful for secure communication. 
TABLE 12: Comparison of MLC for proposed S-box over different S-boxes.

\begin{tabular}{|c|c|c|c|c|c|}
\hline S-boxes & Entropy & Contrast & Correlation & Energy & Homogeneity \\
\hline \multicolumn{6}{|l|}{ Pepper image } \\
\hline Plaintext & 7.5909 & 0.2760 & 0.9383 & 0.1288 & 0.9024 \\
\hline Proposed & 7.9532 & 8.5155 & 0.0079 & 0.0174 & 0.4100 \\
\hline AES & 7.9211 & 7.5509 & 0.0554 & 0.0202 & 0.4662 \\
\hline Ullah et al. [24] & 7.9823 & 8.6727 & -0.0043 & 0.0173 & 0.4076 \\
\hline Skipjack & 7.7561 & 7.7058 & 0.1205 & 0.0239 & 0.4708 \\
\hline Khan et al. [25] & 7.9562 & 8.3129 & 0.0103 & 0.0180 & 0.4219 \\
\hline Belazi & 7.9233 & 8.1423 & -0.0112 & 0.0286 & 0.4648 \\
\hline \multicolumn{6}{|l|}{ Baboon image } \\
\hline Plaintext & 7.1273 & 0.7179 & 0.6782 & 0.1025 & 0.7669 \\
\hline Proposed & 7.9551 & 8.5267 & $4.4609 e-004$ & 0.0174 & .4088 \\
\hline AES & 7.2531 & 7.5509 & 0.0554 & 0.0202 & 0.4662 \\
\hline Prime & 6.9311 & 7.6236 & 0.0855 & 0.0202 & 0.4640 \\
\hline Xyi & 7.2531 & 8.3108 & 0.0417 & 0.0196 & 0.4533 \\
\hline Skipjack & 7.2531 & 7.7058 & 0.1025 & 0.0193 & 0.4689 \\
\hline Khan et al. [25] & 7.9612 & 8.1213 & -0.0512 & 0.0210 & 0.4011 \\
\hline Belazi & 7.9252 & 8.0391 & 0.01190 & .02219 & 0.4428 \\
\hline
\end{tabular}
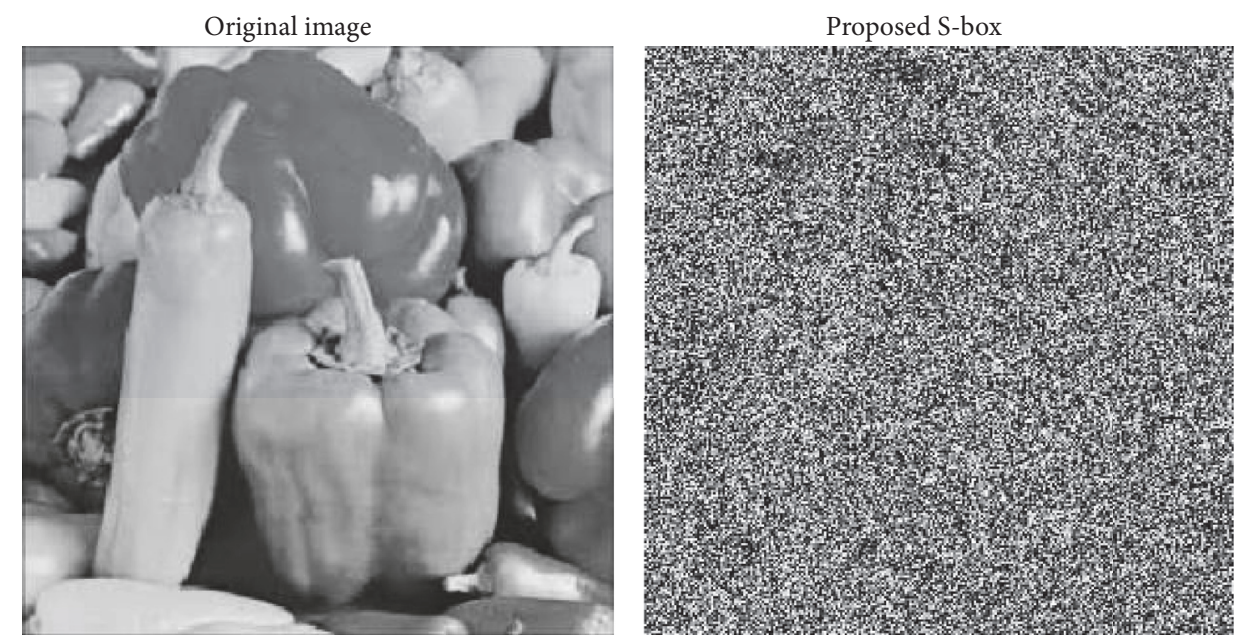

(a)
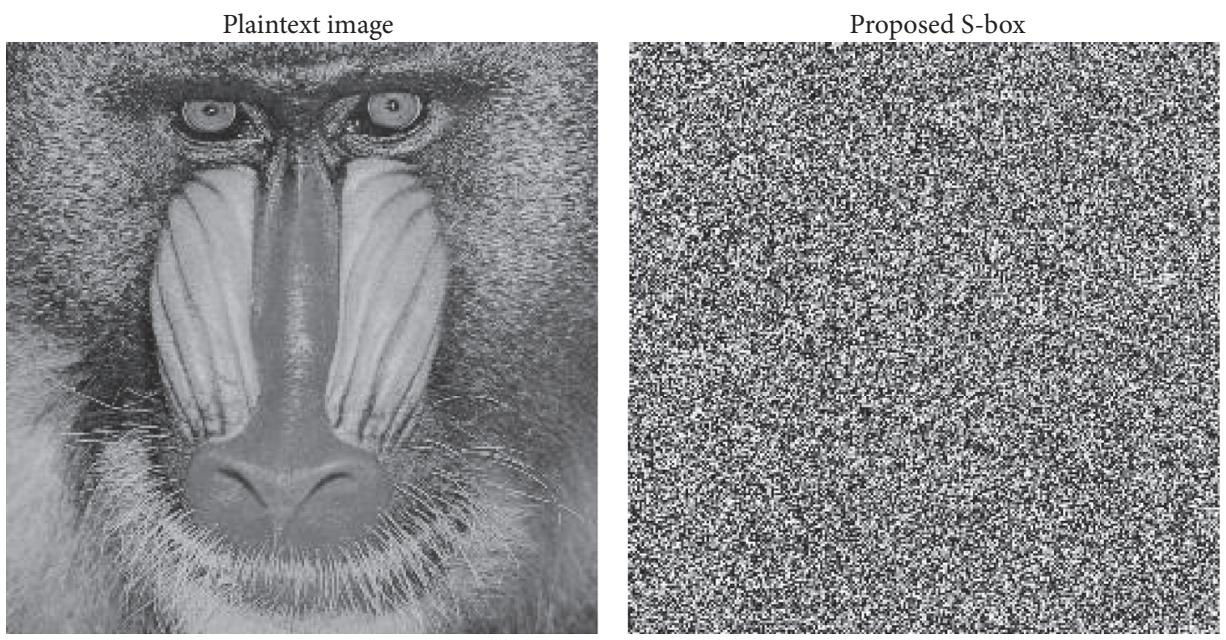

(b)

Figure 6: Original image and the encrypted images using two rounds of encryption: (a) Pepper and (b) Baboon. 

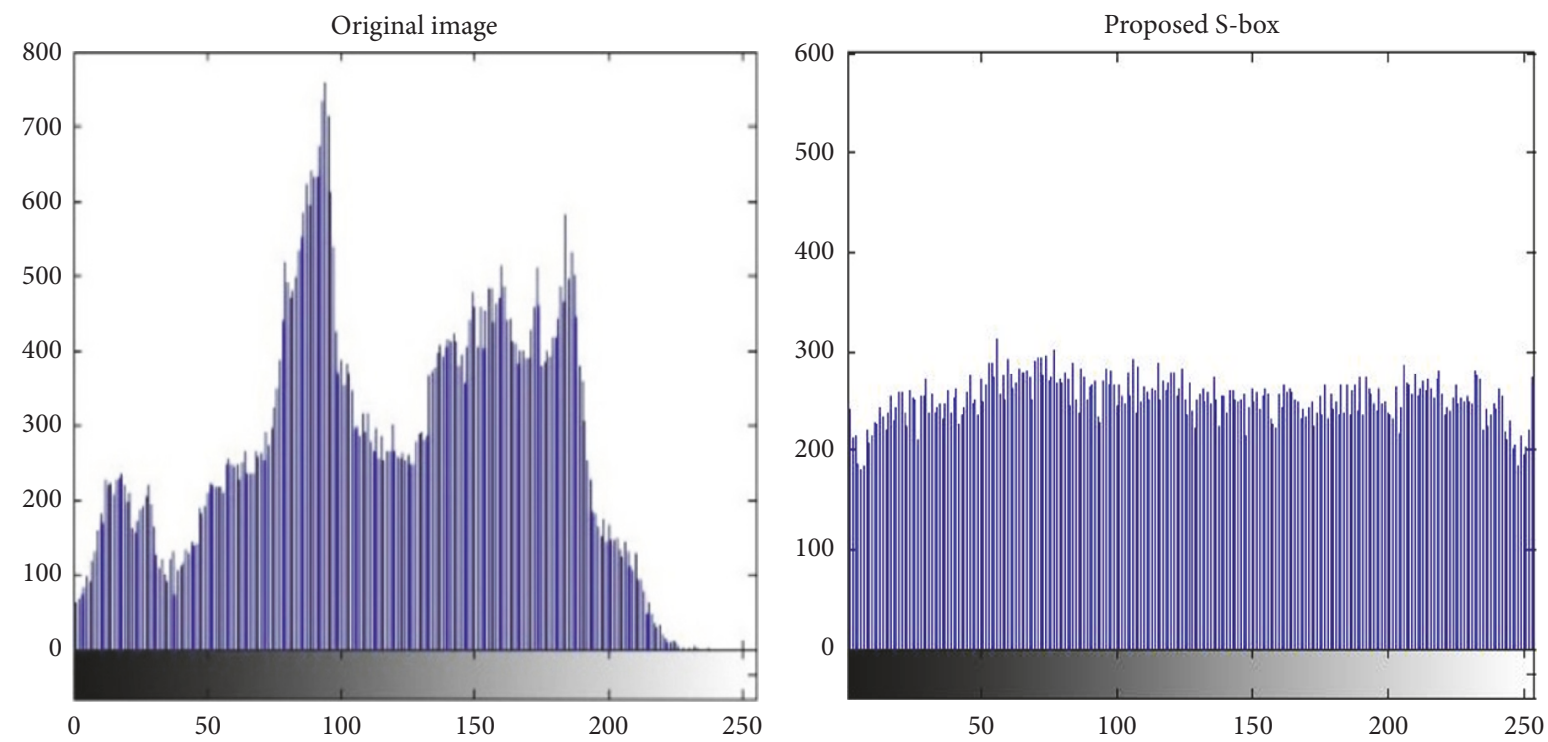

(a)
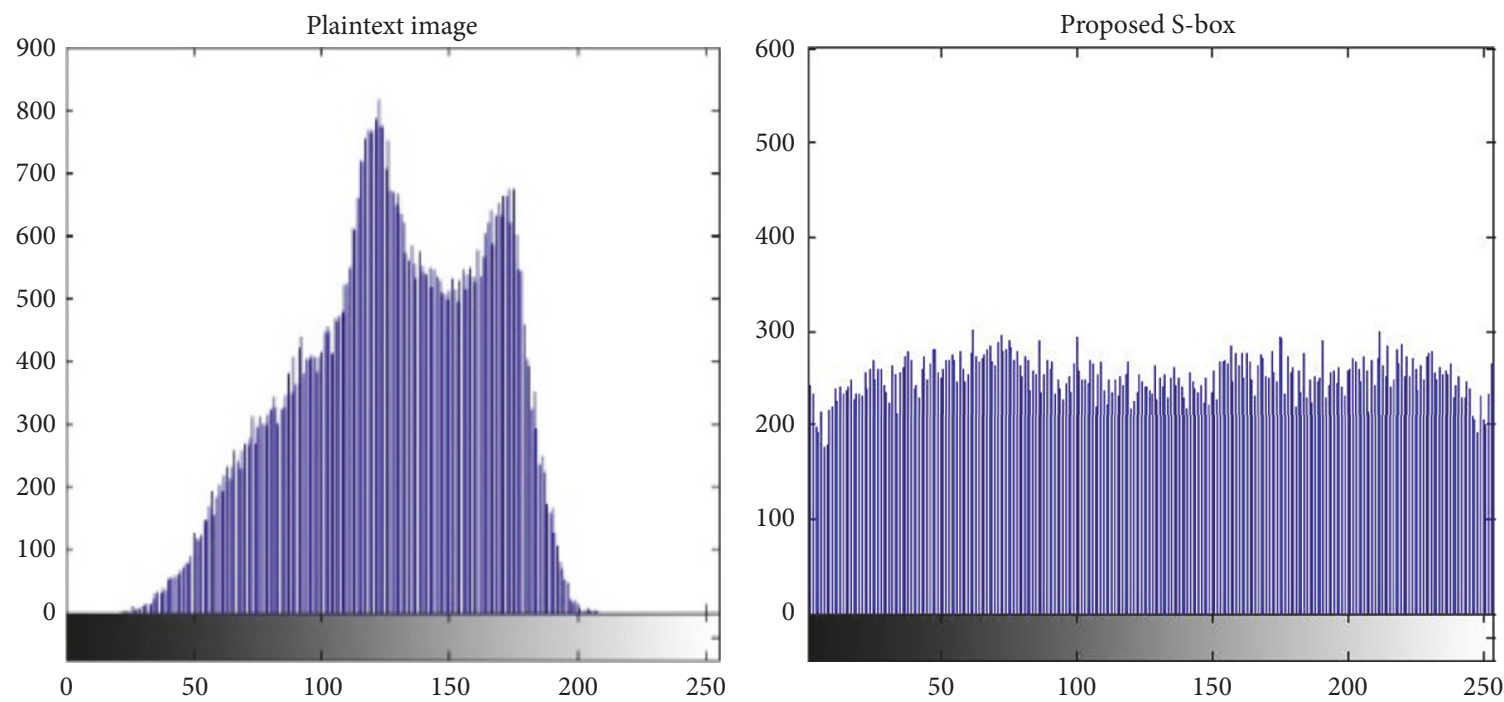

(b)

FIGURE 7: Histogram of the original image and the encrypted images: (a) Pepper and (b) Baboon.

\section{Conflicts of Interest}

The authors declare that there are no conflicts of interest regarding the publication of this paper.

\section{References}

[1] C. E. Shannon, "Communication theory of secrecy systems," Bell Labs Technical Journal, vol. 28, pp. 656-715, 1949.

[2] J. Daemen and V. Rijmen, The Design of Rijndael-AES: The Advanced Encryption Standard, Springer, Berlin, Germany, 2002.

[3] P. J. Cameron, "encyclopaedia of design theory," in Cayley Graphs and Coset Diagrams, pp. 1-9, 2013.

[4] B. Everitt, "Alternating quotients of the $(3, \mathrm{q}, \mathrm{r})$ triangle groups," Communications in Algebra, vol. 25, no. 6, pp. 1817-1832, 1997.
[5] R. C. Lyndon and E. Paul, Combinatorial group theory, vol. 89, Springer, 2015.

[6] Q. Mushtaq and H. Servatius, "Permutation representation of the symmetry groups of regular hyperbolic tessellations," Journal of the London Mathematical Society, vol. 2, no. 48, pp. 77-86, 1993.

[7] A. Torstensson, "Coset diagrams in the study of finitely presented groups with an application to quotients of the modular group," Journal of Commutative Algebra, vol. 2, no. 4, pp. 501514, 2010.

[8] J. Pieprzyk and G. Finkelstein, "Towards effective nonlinear cryptosystem design," IEE Proceedings Part E Computers and Digital Techniques, vol. 135, no. 6, pp. 325-335, 1988.

[9] I. Vergili and M. D. Yücel, "Avalanche and bit independence properties for the ensembles of randomly chosen $\mathrm{n} \times \mathrm{n}$ s-boxes," Turkish Journal of Electrical Engineering \& Computer Sciences, vol. 9, no. 2, pp. 137-145, 2001. 
[10] A. F. Webster and S. E. Tavares, "On the design of s-boxes, advances in cryptology," in Proceedings of CRYPTO'85, Springer, Berlin, Germany, 1986.

[11] E. Biham and A. Shamir, "Differential cryptanalysis of DES-like cryptosystems," Journal of Cryptology, vol. 4, no. 1, pp. 3-72, 1991.

[12] I. Hussain, T. Shah, M. A. Gondal, and H. Mahmood, "Generalized Majority Logic Criterion to Analyze the Statistical Strength of S-Boxes," Zeitschrift für Naturforschung A, vol. 67, no. 5, pp. 282-288, 2012.

[13] M. T. Tran, D. K. Bui, and A. D. Doung, "Gray S-box for advanced encryption standard," in Proceedings of the International Conference on Computer Intel Security, vol. 1, pp. 253-258, 2008.

[14] A. Gautam, G. S. Gaba, R. Miglani, and R. Pasricha, "Application of Chaotic Functions for Construction of Strong Substitution Boxes," Indian Journal of Science and Technology, vol. 8, no. 28, pp. 1-5, 2015.

[15] I. Hussain, T. Shah, H. Mahmood, M. A. Gondal, and U. Y. Bhatti, "Some analysis of S-box based on residue of prime number," Proceedings of the Pakistan Academy of Sciences, vol. 48, no. 2, pp. 111-115, 2011.

[16] I. Hussain, T. Shah, and H. Mahmood, "A new algorithm to construct secure keys for AES," International Journal of Contemporary Mathematical Sciences, vol. 5, no. 25-28, pp. 1263-1270, 2010.

[17] X. Y. Shi, Hu. Xiao, X. C. You, and K. Y. Lam, "A method for obtaining cryptographically strong $8^{\star} 8$ S-boxes," in Proceedings of the International Conference on Advanced Information Networking and Applications, vol. 2, pp. 14-20, 2002.

[18] Skipjack and Kea, “Algorithm Specifications Version 2," http:// csrc.nist.gov/CryptoToolkit/.

[19] A. H. Alkhaldi, I. Hussain, and M. A. Gondal, "A novel design for the construction of safe S-boxes based on TDERC sequence," Alexandria Engineering Journal, vol. 54, pp. 65-69, 2015.

[20] G. Chen, Y. Chen, and X. Liao, "An extended method for obtaining S-boxes based on three-dimensional chaotic baker maps," Chaos, Solitons \& Fractals, vol. 31, no. 3, pp. 571-579, 2007.

[21] G. Tang, X. Liao, and Y. Chen, "A novel method for designing S-boxes based on chaotic maps," Chaos, Solitons \& Fractals, vol. 23, no. 2, pp. 413-419, 2005.

[22] M. Khan, T. Shah, and M. A. Gondal, "An efficient technique for the construction of substitution box with chaotic partial differential equation," Nonlinear Dynamics, vol. 73, no. 3, pp. 1795-1801, 2013.

[23] A. Belazi, M. Khan, A. A. A. El-Latif, and S. Belghith, "Efficient cryptosystem approaches: S-boxes and permutationsubstitution-based encryption," Nonlinear Dynamics, vol. 87, no. 1, pp. 337-361, 2017.

[24] A. Ullah, S. S. Jamal, and T. Shah, "A novel construction of substitution box using a combination of chaotic maps with improved chaotic range," in Nonlinear Dynamics, vol. 88, pp. 2757-2769, Dynamics, 2017.

[25] M. Khan, T. Shah, and S. I. Batool, "Construction of S-box based on chaotic Boolean functions and its application in image encryption," Neural Computing and Applications, vol. 27, no. 3, pp. 677-685, 2016. 


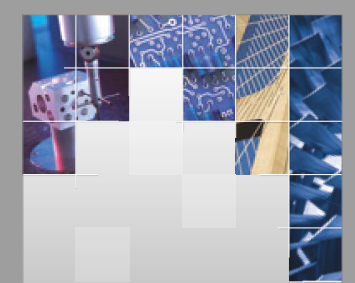

\section{Enfincering}
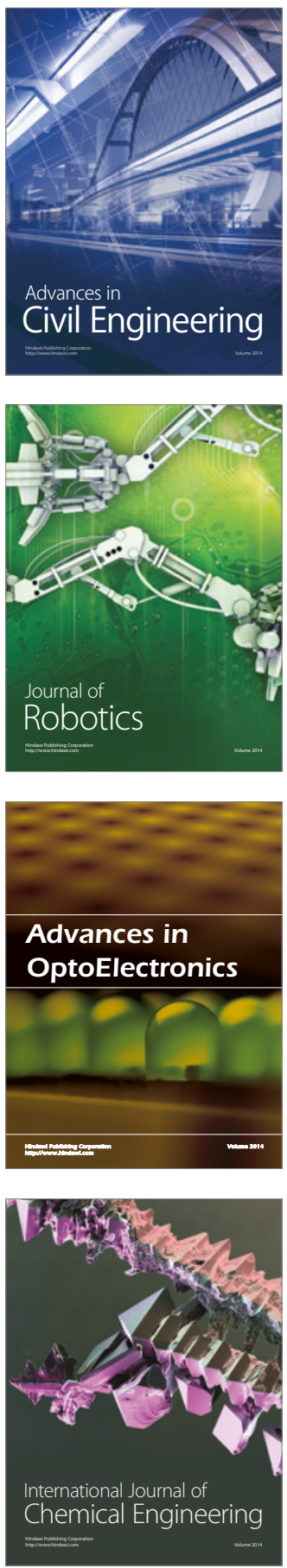

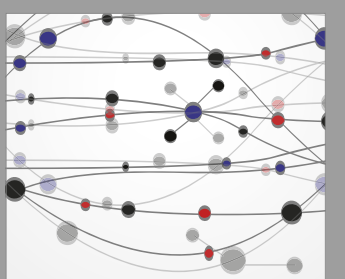

The Scientific World Journal

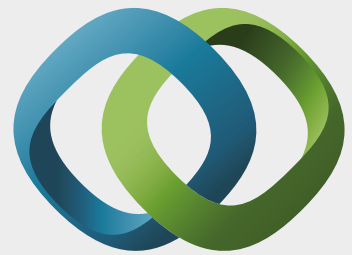

\section{Hindawi}

Submit your manuscripts at

https://www.hindawi.com
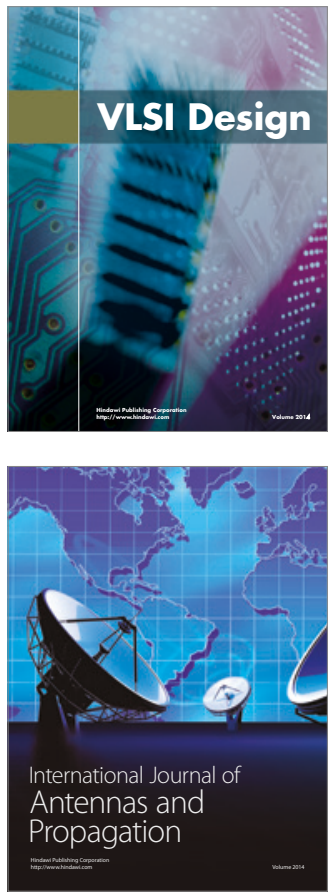

\section{Rotating}

Machinery
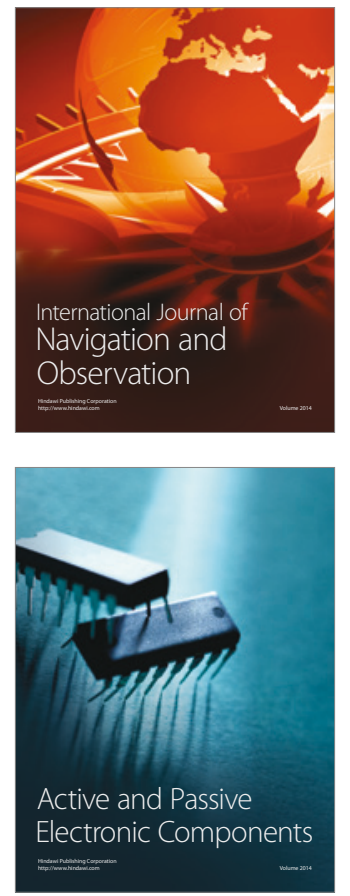
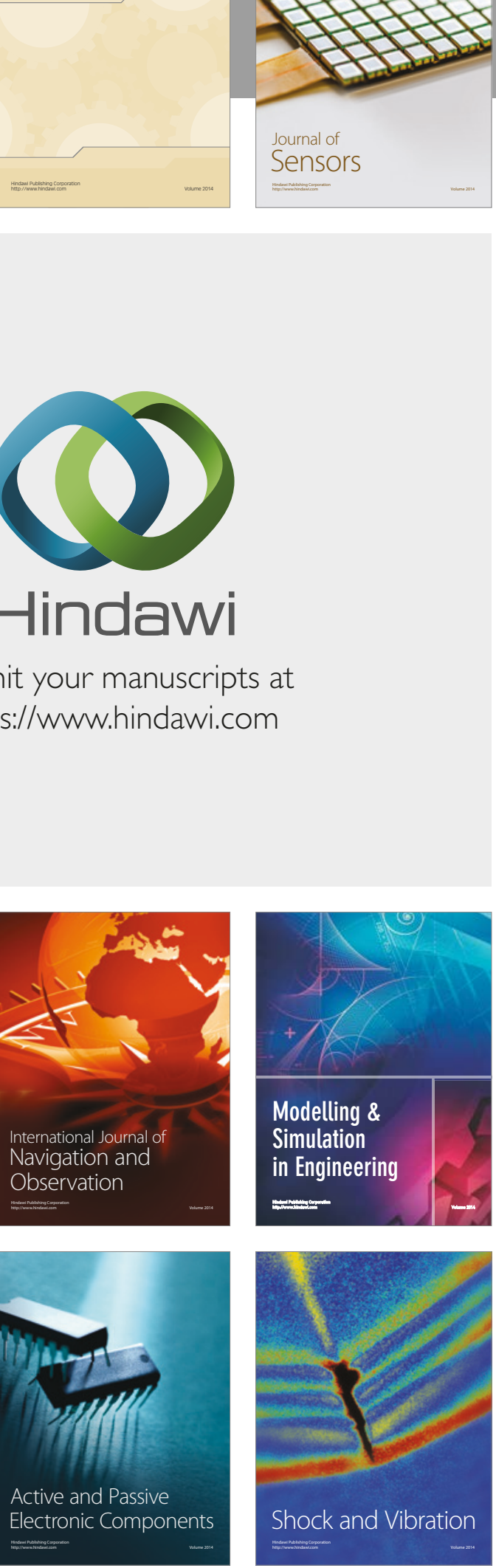
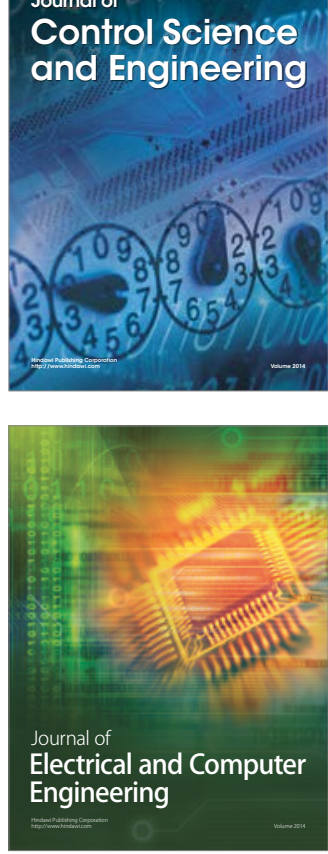

Distributed

Journal of

Control Science

and Engineering
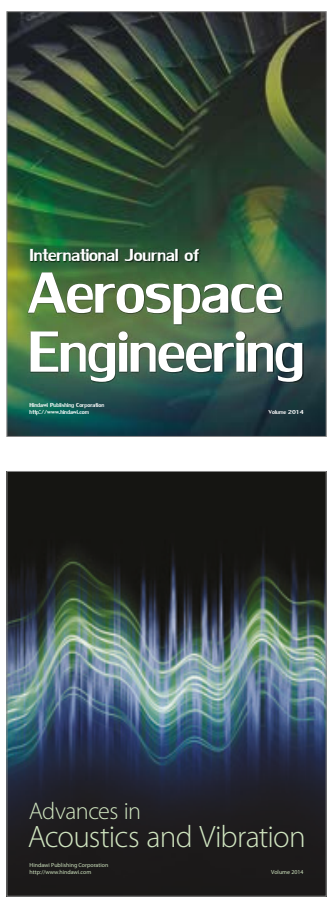

Sensor Networks 